\title{
Assessing Effects of Life History Antecedents on Age at Menarche and Sexual Debut Using a Genetically Informative Design
}

\author{
Nicole Barbaro ${ }^{1 *}$, George B. Richardson², Joseph L. Nedelec ${ }^{3}, \&$ Hexuan Liu ${ }^{3}$ \\ ${ }^{1}$ Oakland University, Department of Psychology \\ ${ }^{2}$ University of Cincinnati, School of Human Services \\ ${ }^{3}$ University of Cincinnati, School of Criminal Justice
}

Word count (all inclusive): 13,076

*Corresponding author: Nicole Barbaro, Oakland University, Department of Psychology, 654 Pioneer Drive, Rochester MI, 48309. Email: n.barbaro.psych@gmail.com

George B. Richardson, University of Cincinnati, School of Human Services, PO Box 210002, Cincinnati OH, 45221.Email: george.richardson@uc.edu

Joseph L. Nedelec, University of Cincinnati, School of Criminal Justice, PO Box 210389, Cincinnati, OH, 45221.

Email: joseph.nedelec@uc.edu

Hexuan Liu, University of Cincinnati, School of Criminal Justice, PO Box 210389, Cincinnati, OH 45221. Email: Hexuan.liu@uc.edu 


\begin{abstract}
(120)
Life history derived models of female sexual development all propose menarche timing as a key regulatory mechanism driving subsequent sexual behavior. The current research utilized a twin subsample of the National Longitudinal Study of Adolescent to Adult Health (Add Health; $n=520$ ) to comprehensively evaluate effects of life history antecedents on menarche timing and sexual debut, and extend understanding of these life history models with a genetically informative design. The results show mixed support for each life history model and did not provide evidence any effect on age at menarche. Results of this research call to question the underlying assumptions of life history derived models of sexual development and highlight the need for more behavior genetic research in this area.
\end{abstract}

Keywords: Age at menarche; life history theory; psychosocial acceleration; father absence; BMI; genetic confounding; Add Health; sexual development. 


\section{Assessing Effects of Life History Antecedents on Age at Menarche and Sexual Debut Using a Genetically Informative Design}

Life history theory (LHT) is a biological framework that explains differences between species in terms of fitness trade-offs imposed upon organisms (e.g., offspring quantity vs. quality, mating vs. parenting effort, and current vs. future reproduction; Hill \& Kaplan, 1999; Stearns, 1992). In the absence of constraints, selection would presumably maximize investment in all fitness components (Agrawal, et al., 2010). However, given that resources are limited, species vary in developmental tempo, reproductive timing, offspring number, body size, and longevity (Stearns, 1976).

LHT was extrapolated to variation within humans nearly 40 years ago (Draper \& Harpending, 1982), with many applications focused on the fundamental trade-off between current and future reproduction in which allocation of resources to early reproduction is expected to occur at the expense of continued bodily growth and maintenance, whereas continued growth and development is expected to incur costs associated with delaying reproduction (Kaplan \& Gangestad, 2005). This trade-off is utilized to explain variation in developmental trajectories across the life span (Del Giudice et al., 2015). In particular, it can be leveraged to explain individual differences in indicators of reproductive schedule such as pubertal timing, age at sexual debut, and age at first birth (Kaplan \& Gangestad, 2005).

Under high levels of extrinsic mortality (i.e., local risk of death that is not accounted for by allocation of effort to fitness components), organisms are more likely to lose investments in somatic and parental effort (Placek \& Quinlan, 2012; Quinlan, 2010). Applications of LHT within the field of developmental psychology have therefore predicted that humans tend to invest more in early reproduction and mating effort, at the expense of somatic and parent effort, in response to early extrinsic mortality cues (Ellis et al., 2009). Because experiences with primary caregivers mediate the degree of extrinsic environmental risk, especially early in development, early rearing experiences are posited to influence the timing of reproductive milestones (e.g., Belsky et al., 1991; Draper \& Harpending, 1982; Ellis, 2004).

In the first application of LHT to human development, Draper and Harpending (1982) proposed paternal investment theory, focusing specifically on the impacts of male investment in offspring. Paternal investment theory stipulates that the degree of paternal investment experienced provides important ecological and relational 
information to the offspring that should influence pubertal maturation timing and subsequent sexual strategies in adolescence and adulthood. Father absence, or a lack of male investment in early development, is presumed to signal to the offspring that male investment is unavailable, and unnecessary. Such cues are assumed to be predictive of the adult environment and, given that patemal investment from a mate in adulthood is unlikely, it is therefore advantageous for individuals to achieve sexual maturity earlier and allocate greater proportion of resources to early reproduction (Webster et al., 2014).

Belsky, Steinberg, and Draper (1991) later expanded paternal investment theory to include a broader array of indices that might signal environmental risk in early development, of which father absence remains an important cue. Psychosocial acceleration theory (Belsky et al., 1991) proposes that environmental stress early in development - with foci on fragmented family structures, inadequate household resources, parenting styles, extrinsic mortality risk - accelerates reproductive development (see also, Chisholm et al., 1993; Ellis et al., 2009). Consistent with this reasoning, studies find that individuals who experience relatively harsh or unpredictable rearing environments are more likely to achieve sexual maturity earlier (Ellis, 2004), engage in (risky) sexual behaviors at a younger age (Belsky et al., 2012; Simpson et al., 2012), and have less stable or shorter romantic relationships in adulthood (Del Giudice, 2009; Simpson et al., 2011). Like paternal investment theory (Draper \& Harpending, 1982), psychosocial acceleration theory is generated from the fundamental trade-off between early and future reproduction.

Finally, child development theory (Ellis, 2004) offers subtly different predictions about the connections between early environmental stress, menarche timing, and subsequent reproductive behavior. Consistent with paternal investment theory (Draper \& Harpending, 1982) and psychosocial acceleration theory (Belsky et al., 1991), child development theory proposes that early environmental stress accelerates menarche timing as a means to optimally adjust the duration of childhood as a function of local ecology (Ellis, 2004). Child development theory, however, differs from the other life history models of pubertal timing in that it makes no predictions connecting menarche timing to subsequent reproductive strategies, including sociosexual orientation or number of sexual partners. Ellis (2004) proposes that menarche timing plausibly only predicts age at sexual debut and reproductive timing simply because such reproductive traits are natural sequelae of pubertal development; menarche timing therefore has no theoretical connection to sexual strategies in adulthood from this perspective. 
Life history models of development have often investigated antecedents and sequelae of pubertal timing because it is a universal and key phase in sexual development (e.g., Belsky, 2019; Ellis, 2004). Life history developmental models are underscored by a number of core assumptions affording generation of hypotheses about pubertal timing: (1) cognitive and behavioral plasticity permits conditional adjustment of reproductive strategies, such that early experiences are capable of calibrating the timing of sexual maturity and reproductive behavior best aligned to the local environment (Bjorklund, 2016; Del Guidice, 2015); (2) reproductive traits covary with particular ecological features, partly because humans are thought to accelerate reproductive development in response to cues to harshness and unpredictability (Ellis et al., 2009); (3) reproductive traits are interrelated, such that investment in current reproduction manifests as both earlier menarche timing and age at sexual debut, as well as trades off against investment in components of future reproduction (Figueredo et al., 2006).

Together, these assumptions underpin core tenets of life history derived developmental models for explaining variation of menarche timing and reproductive outcomes in girls (Belsky et al., 1991; Draper \& Harpending, 1982; Ellis, 2004). Research generated from these developmental models of menarche timing, for example, generally support these three assumption siven that: (1) earlier menarche is often associated with other reproductive outcomes, most notably, age at sexual debut (Copeland et al., 2010; Ibitoye et al., 2017); (2) earlier menarche timing is associated with greater environmental risk (Belsky et al., 1991; Ellis et al., 2009); and (3) environmental stress, including father absence, in early development is associated with earlier menarche timing, consistent with the notion of facultative adjustment of sexual maturity as a function of early experiences (Belsky, 2019; Ellis, 2004; Webster et al., 2014).

\section{Research Aims}

Aspects of life history developmental models of pubertal timing, described above, have been subject to recent debate. Much of the debate has focused on the association between early rearing environments and reproductive outcomes (see point 3, above); in particular the degree to which reproductive strategies correspond with features of the early rearing environment, and the degree to which variation of menarche timing is a result of facultative adjustments in response to early environmental cues. Two specific criticisms within this literature have been featured in this debate: (1) father absence, or fragmented family structure more generally, might be 
confounded by other environmental factors such as socioeconomic status (Richardson et al., 2018) or caloric intake (Kyweluk et al., 2018), especially in WEIRD societies (i.e., Western, Educated, Industrialized, Rich, Democratic; Sear et al., 2019); and/or (2) the small, presumably causal effect, of environmental stress broadly, and father absence specifically, on menarche timing might largely be a result of common genetic influences on the traits of interest, or what is commonly referred to as "genetic confounding" (Barbaro et al., 2017; Rowe, 2002; which can masquerade as an environmental or rearing effects in non-genetically informative study designs).

Although each of these criticisms and alternative explanations for menarche timing have been investigated independently in previous research (e.g., Barbaro et al., 2017; Kyweluk et al., 2018; Richardson et al., 2018; Sear et al., 2019), no previous study has attempted to comprehensively evaluate a broad array of proposed effects of life history antecedents on menarche timing (that are derived from life history developmental models) with a genetically informative research design. The current research utilizes a twin subsample of The National Longitudinal Study of Adolescent to Adult Health (Add Health) to evaluate menarche antecedents (e.g., socioeconomic status, family structure, body mass index) and consequences (e.g., sexual debut, risky sexual behavior). Below, we outline each of our specific research aims.

\section{Assess the association between father absence, specifically, and age at menarche while controlling for familial structure and socioeconomic status}

A meta-analysis of 33 studies indicates that father absence has a small, yet reliable association with earlier menarche (Webster et al., 2014). A limitation of the meta-analysis, however, is that potentially confounding environmental effects were not considered (Davis \& Friel, 2001; Richardson et al., 2018). According to psychosocial acceleration theory (Belsky et al., 1991) and child development theory (Ellis, 2004), generally stressful environments are hypothesized to accelerate development. Children residing in father absent households, due primarily to parental separation and abandonment, may be at increased risk for experiencing other stres sors such as unrelated male presence (e.g., step fathers), lower income, neglect and abuse — which have been linked previously to earlier age at menarche (e.g., Boynton-Jarrett et al., 2013; Romans et al., 2003). It is therefore reasonable to suspect that previously reported father absence effects may rather be mediated by stress or due to confounding familial stressors that commonly co-occur with father absence. Research by investigating the influence of familial structure on age at menarche has found some evidence in support of the father absence effect 
(Richardson et al., 2018) as conceptualized by paternal investment theory (Draper \& Harpending, 1982), documenting evidence consistent with a small, accelerating effect of temporary father departure (i.e., $>1$ year) in intact families that survives controls for broader family structure. In contrast, Richardson et al. (2018) found no evidence that fragmented family structure was related to age at menarche, as conceptualized by psychosocial acceleration theory (Belsky et al. 1991). In the current study, a father absence index is created to more comprehensively quantify the duration of father absence experienced, while controlling for additional components offamilial structure to provide a robust test of the impact offather absence and family structure on age at menarche.

\section{Assess the association between father military service and age at menarche}

The classical conceptualization of father absence from a paternal investment theoretical perspective is that of separation and abandonment, both of which are directly related to the extent of resource investment into the child. Other reasons for father absence, however, may be expected to also accelerate menarche timing (Davis \& Friel, 2001; Richardson et al., 2018; Shenk et al., 2013). Military service is often associated with temporary father departure, in which regular contact between father and child may be severely disrupted, in addition to a decrease in direct investment from the father. Military service can also cause stress on the spouse, which may indirectly affect matemal investment and sensitivity, especially to young children, as well as cause disruption of peerrelationships and schooling because of moving between bases. Impacts of military service would arguably be encompassed by psychosocial acceleration theory to the extent that military service covaries with perceptions of predictability, which has been shown to be associated with accelerated menarche and sexual behavior (Belsky et al., 2012; Simpson et al., 2012). The current study assesses the unique association of fathers' military service with age at menarche as an initial attempt to investigate whether military service functions similarly to classical conceptualizations of father absence and psychosocial stressors.

\section{Assess the association between father-figure genetic relatedness and age at menarche}

Another important aspect of the father absence effect deserving of empirical attention is children growing up with unrelated males in the household, in that the absence of the biological father increases the probability of an unrelated male residing in the household. As suggested by Belsky et al. (1991), the absence of the biological father may be signaling a lower risk of inbreeding, rather than a lack of paternal investment. Put differently, 
mechanisms facilitating plasticity of menarche timing may be responding to cues to genetic relatedness of males in the household, rather than the absence of paternal investment, specifically. Genetic relatedness of the primary male of the household may therefore, in part, account for the father absence effect given that inbreeding risk is lower in father absent households (Matchock \& Susman, 2006). Evidence of this alternative account for the father absence effect in humans has to our knowledge not been presented, and evidence in non-humans is mixed (Blouin \& Blouin, 1988; Charpentier et al., 2008; Pusey \& Wolf, 1996). The current study investigates this possibility by assessing the association between the genetic relatedness of the father-figure in the household, while controlling for other aspects of family structure.

\section{Assess the direct association between birthweight and age at menarche, and the indirect association via body mass index (BMI)}

Somatic growth is dependent on energy intake. A fundamental prediction from life history theory is that greater energy intake will accelerate growth and, therefore, pubertal timing (Kuzawa \& Bragg, 2012; Promislow \& Harvey, 1990). Age at menarche in girls has been decreasing world-wide, with declining rates of poverty and greater access to food being proposed as primary contributing factors (Parent et al., 2003). Age at menarche is lowest in the most developed and industrialized nations, such as the United States, presumably a result of substantial decreases in health insults. In the United States, where the majority of research on psychosocial acceleration theory has been conducted (see Sear et al., 2019), socioeconomic status is often confounded by excess weight and caloric abundance (Kyweluk et al., 2018; Sohn, 2017). Because of these confounds, effects of father absence and other markers of socioeconomic stress in the United States and other WEIRD countries may appear significant (see Webster et al., 2014), but are likely to be inflated (Kuzawa \& Bragg, 2012). In support of this notion, studies in countries without caloric overabundance show no support for effects of psychosocial stress and familial instability on menarche timing (Anderson, 2015; Kyweluk et al., 2018; Sheppard et al., 2014; Sear et al., 2019; Sohn, 2017). The current study will therefore investigate the associations between birthweight and BMI on age at menarche, while controlling for effects of socioeconomic status and fragmented family structure.

\section{Assess direct association between family structure and sexual debut, and the indirect association via age at menarche}


The fundamental life history trade-off between current and future reproduction (Del Giudice et al., 2015) suggests that earlier maturation should facilitate earlier age of sexual debut (see La Guardia et al., 2014). Although a seemingly straightforward prediction of life history developmental models of sexual development (Ellis, 2004), few studies have tested the temporal sequence of environmental stress on sexual debut via age at menarche. To date, three studies have provided some evidence consistent with a mediational pathway from environmental stress to sexual debut via age at menarche (Belsky et al., 2010; James et al., 2012; Richardson et al., 2018). The current study provides an additional test of the mediational pathway predicting sexual debut via age at menarche, while controlling for aspects of socioeconomic status and somatic growth.

\section{Assess associations between sexual debut and reproductive outcomes}

A primary assumption underpinning life history research is that reproductive traits covary. This covariance of traits is referred to as life history strategies (Figueredo et al., 2006). Life history strategies reflect coordinated patterns of phenotypic traits (Braendle et al., 2011) that are often situated conceptually on a slow-fast continuum (Promislow \& Harvey, 1990; $c f$. Richardson et al., 2017a, b). Earlier menarche and age at sexual debut constitute a relatively fast life history strategy favoring resource allocation to current reproduction at the expense of prolonged somatic growth. A fast life history strategy is additionally characterized by more risky sexual behavior, greater number of sexual partners, and a greater desired number of offspring (Del Giudice, 2009; Ellis et al., 2012; Simpson et al., 2012). In accordance with psychosocial acceleration theory (Belsky et al., 1991), the current study investigates the associations between sexual debut and reproductive strategies, hypothesizing that earlier sexual debut will be associated with more risky sexual behavior (e.g., engaging in sexual intercourse under the influence of alcohol, unprotected sexual intercourse), greater number of sexual partners, and greater desired number of children.

\section{Assess for genetic confounding of associations}

Decades of behavioral genetic research shows that complex phenotypic traits are heritable (Polderman et al., 2015), including traits that comprise life history strategies (Figueredo et al., 2004) and features of home environments (Kendler \& Baker, 2007). To the extent to which genetic variation underpinning early experiences and family environment covary with genetic variation underpinning reproductive traits, life history developmental hypotheses are susceptible to genetic confounding (Barbaro et al., 2017; Rowe, 2002). Put differently, purportedly 
causal pathways between early experiences and pubertal timing may be attributable to common genetic influences, which may render (especially small) reported environmental effects largely spurious. Results of investigations attempting to parse out genetic influences to assess the robustness of effects of life history antecedents on menarche timing are mixed and use varying designs. Some investigations have shown no effect of father absence on menarche once genetic variation is accounted for (D’Onofrio et al. 2006; Mendle et al., 2006), whereas other investigations have not found evidence to support the genetic confounding hypothesis using sibling (Tither \& Ellis, 2008), candidate gene (Schlomer et al., 2019) or polygenic score approaches (Gaydosh et al., 2018). Each of these investigations, however, include only a narrow set of environmental or genetic variables. Conclusions with regard to the robustness of life history developmental models against genetic controls therefore remain limited. The current study uses a genetically informed design to assess the extent to which genetic factors account for associations detected in a comprehensive structural equation model (SEM).

\section{The Current Study}

To achieve the research aims outlined above, we employed a two-stage analytic approach and data from a community sample of female twins. Our approach was designed to address potential confounding of effects along the cascade from early environment to life history events such as menarche timing and sexual debut. The two stages allowed for comparison of estimates from models that did and did not account for potential genetic confounding, thereby providing information about the utility of adopting a genetically informative design as well as clearly linking our genetically informative findings back to prior research. In the first stage, we used structural equation modeling (SEM) to build a comprehensive model of life history antecedents - including father absence, family structure, and socioeconomic status - to predict age at menarche and sexual debut. This initial stage represented an attempt to replicate findings from a student sample reported by Richardson et al. (2018) and mirrored standard practice in that the modeling approach was non-genetically informative. Stage one moved beyond past research, however, by including a broader suite of life history antecedents and sequelae of menarche, which in turn permitted more critical testing of paternal investment, psychosocial acceleration, and child development theories. See Figure 1 for a high-level conceptual model of the predictions that are tested in the present research. 
In the second stage, we used a genetically informative design to address potential genetic confounding of environmental effects detected in our non-genetically informative SEM. In brief, behavioral genetics is a field in which phenotypic variance of a trait of interest is composed of three components: heritability $\left(h^{2}\right)$, shared environment $\left(c^{2}\right)$, and nonshared environment $\left(e^{2}\right)$. The $h^{2}$ component refers to phenotypic variance accounted for by variance in genes. The environmental components $\left(c^{2}\right.$ and $\left.e^{2}\right)$ refer to phenotypic variance accounted for by environmental experiences shared with others (i.e., aspects of the environment that make people similar to one another, $c^{2}$ ) and environmental experiences not shared with others (i.e., aspects of the environment that make people dissimilar, $e^{2}$ ). These three components account for 100 percent of the variance in any phenotype. There are a number of methods used to decompose phenotypic variance, but classical twin-based designs that compare dizygotic (DZ) twins to monozygotic (MZ) twins are most common. Researchers have repeatedly shown that these designs are theoretically and methodologically sound (Barnes et al., 2014; Wright et al., 2015). In addition to decomposition of variance models, behavioral genetics includes a variety of methodologies that can be employed to produce more plausible estimates of causal effects via control of potentially confounding genetic and shared environmental factors (Johnson et al., 2009; McGue et al., 2010; Turkheimer \& Harden, 2014). By controlling measured environment, as well as determining the relative importance of unmeasured genetic and environmental factors, we were able to subject paternal investment, psychosocial acceleration, and child development theories to more stringent testing than prior non-genetically informative studies.

\section{Method}

\section{Preregistration}

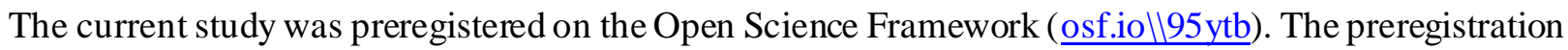
includes: (1) a hypothesized measurement model of early stress factors; (2) a hypothesized SEM; (3) research aims 1-7 as described above; (4) a table of measures from the Add Health used in the current study; and (5) an analysis plan. Deviations from our preregistered plan are noted in the Methods and Results in the main text, with accompanying details and rationale provided in footnotes.

\section{Data}

The current study used the restricted data from the National Longitudinal Study of Adolescents to Adult Health (Add Health; Harris et al., 2006). Details regarding the sampling procedures and instruments are described 
in detail elsewhere (see Harris et al., 2006). Briefly, the Add Health is a longitudinal prospective study of a nationally representative sample of youth in America interviewed at five different time points over the course of approximately 25 years (Wave 1: 1994-95; Wave 2: 1996; Wave 3: 2001-02; Wave 4: 2008; Wave 5: 2016-19). The first two waves of interviews included questions pertaining to adolescence as the majority of respondents were between ages 14 and 18 years. Questionnaires were altered in waves 3 and 4 as the respondents were in their early twenties and at or beyond the age of 25, respectively (approximately $20 \%$ of the respondents were aged 30 years or older at Wave 4). At Wave 5, questionnaires were again altered as the ages of respondents ranged from 33 to 44 . The current study employs data from the first four waves of interviews (Wave 5 was not available at the time analyses were conducted). Given that the data are secondary and de-identified, the Institutional Review Board at the University of Cincinnati determined that the data did not meet the regulatory criteria for research involving human subjects.

The Add Health was meant to be a nationally representative sample, but there were specific additional sampling procedures that took place during the first wave of data collection. Pertinent to the current study, twins, siblings, and other kinships (e.g., cousins) of the original target respondents were oversampled (i.e., guaranteed enrollment in the sample; Harris et al., 2006). Nested within the over 3,000 kinship pairs were 307 monozygotic (MZ) and 452 dizygotic (DZ) twin pairs (zygosity was assessed using genotype data; see Harris et al., 2006). ${ }^{1}$ The current study employed data from the female twin pairs. Due to the exclusion of opposite-sex DZ twins, the analytical sample was comprised of $143 \mathrm{MZ}$ twin pairs $(n=286$ individuals $)$ and $114 \mathrm{DZ}$ twin pairs $(n=228$ individuals), for a total of 257 twin pairs ( $n=514$ individuals). ${ }^{2}$

\section{Measures}

Drawing on substantive theory and prior research (e.g., Richardson et al., 2018), we preregistered—prior to handling the data - a selection of items from the Add Health survey that captured participants' background characteristics, sources of early environmental stress, family structure, socio-economic status (SES), and the pace

\footnotetext{
${ }^{1}$ Although oversampled, prior research has shown that the twin subsample of the Add Health does not differ substantially from the nonsibling sample on a variety of measures (e.g., Barnes \& Boutwell, 2013; Jacobson \& Rowe, 1998; Nedelec et al., 2017).

2 Our preregistered sample size included 254 twin pairs. We ultimately elected to include data from three additionaltwins with no co-twin data, bringing the number of pairs to 257.
} 
of reproductive development (e.g., age at menarche). Post-registration, we inspected the survey and data more closely and determined it was necessary to exclude several items. First, we could not determine if the exposures assessed by some items occurred prior to puberty. ${ }^{3}$ Second, we excluded the desired number of children item (H4MH9) because it did not isolate desired number of children from current number of children. Third, we excluded relation of father figure to participants (H4WP27) because there were not enough levels of genetic relatedness among father figures. ${ }^{4}$ Four, we excluded father military service because parents of only six twin pairs endorsed the item(PA27B) and it was limited to those who were members of veteran's organizations. Finally, we excluded father receipt of public assistance (H1RF9) because only seven fathers had reportedly received it. The remaining items, which we analyzed, are described below and their contents and response options can be found via the Add Health website (https://www.cpc.unc.edu/projects/addhealth).

Descriptive statistics for the twin 1 life history and environmental stress indicators included in Step 1 of the analysis are displayed in Table 1, as are descriptive statistics for the twin 2 life history indicators included in Step 2. We describe construction of indexes measuring several constructs below. Wherever principle components analysis (PCA) was used to construct indexes, we saved scores using Bartlett's method ${ }^{5}$. Unless otherwise noted, indexes were pairwise or computed for both twins. Bivariate MZ and DZ correlations among all variables analyzed are displayed in Supplementary Materials.

Body mass index. Following standard calculations, we computed body mass index (BMI) by dividing Wave 1 weight (H1GH60) by height squared (H1GH59A) and multiplying by 703.

Adverse childhood experiences. We used six items from Waves 1, 3, and 4 to assess twin 1 adverse childhood experiences including age when father died (H1NF3), age when mother died (H1NM3), age biological dad was sent to jail or prison (H4WP11), age biological mom was sent to jail or prison (H4WP5), number of times being hit or kicked by sixth grade (H3MA3), and number of times removed from home by social services by sixth grade (H3MA5). We used age at menarche to recode the age at parental death and age at parent sent to

\footnotetext{
3 These were, "how often in the last month have you (parent) had five or more drinks on one occa sion" (PA62), "how many other states or countries have you lived since June 1995" (H3HR20), "since the beginning of June 1995 at how many (other) addresses have you lived" (H3HR18).

${ }^{4}$ Specifically, $60.5 \%$ were raised by biological fathers, $14.5 \%$ were raised by unrelated father figures, and less than $6.0 \%$ were raised by any type of non-biological but related fatherfigure (i.e., grandfather, brother, uncle, or other male relative.)

${ }^{5}$ Missing values were saved for participants who were missing data on the indicators analyzed with PCA. Missing data were handled via pairwise present in the SEM analyses.
} 
jail or prison items into ever had these experiences prior to puberty. We then summed the resulting items to create an index of early adversity. Twenty-nine and two tenths percent had at least one early adverse experience.

Childhood sexual abuse. We used four variables from Waves 3 and 4 to assess twin 1 childhood sexual abuse. These were age first touched sexually (H4MA6), age first time non-physical forced sex (H4SE33), age first time physical forced sex (H4SE35), and number of times touched in sexual way by sixth grade (H3MA4). We used age at menarche to recode the first three variables into three ever exposed before puberty variables. A small proportion $(6.5 \%)$ had been touched in a sexual way by sixth grade and three of the five response categories, which ranged from "one time" to "more than ten times," had sparse counts. Thus, we recoded this variable into ever touched in a sexual way by sixth grade. Finally, we recoded the resulting variables into ever experienced early sexual abuse. Eight and a half percent of target twins had experienced early sexual abuse.

Father absence. We used three items to assess father absence including years of biological father absence prior to Wave 1 interview (calculated by subtracting the most recent year the target lived with non-resident biological father [PC8; parent interview] from the interview year at Wave 1), years of biological father absence prior to menarche (calculated by subtracting age last lived with non-resident biological father [H1NF8] from age of menarche at Wave $2[\mathrm{H} 2 \mathrm{FP} 4]^{6}$ ), and length of biological father absence (calculated by subtracting age last lived with non-resident biological father [H1NF8] from age at Wave 1). Because these variables were highly correlated ( $r s=.67$ to .91 ), we used PCA to construct a father absence index. We found a single component that explained $82 \%$ of the variance and loadings ranged from .83 to .95 .

Neighborhood quality. We used four items from the parent survey to construct a neighborhood danger index comprised of feels safe in neighborhood (S62Q), moved to neighborhood because it has less of a drug problem (A34), moved to neighborhood because it has less crime (PA28E), and moved to neighborhood because it has less drug use (PA28F). A single component explained 50\% of the variance and loadings ranged from .67 to .89. Higher scores implied parents found their current neighborhood safer and more ideal than others.

Risky behavior. We used four items to construct a risky behavior index, including the number of times participants had sex with more than one partner at the same time (H4SE27), the number of partners they had sex

\footnotetext{
${ }^{6}$ Age of menarche at Wave 2 [H2FP4] was employed to reduce missingness; additionally, respondents who reached age of menarche prior to fatherabsence were coded as " 0 " on this item.
} 
with on only one occasion (H4SE25), drinking alcohol in a way that put them at risk (H4TO47), and using marijuana in a way that put them at risk (H4TO77). A single component explained $38 \%$ of the variance and loadings ranged from .47 to .67 . Higher scores implied greater liability to risky behaviors.

\section{Analytic Approach}

This study used a two-step SEM approach to test for effects of father absence and other aspects of early environment on the pace of female reproductive development. As described previously, in the first step we used covariate control to address potential confounding of father absence and early environmental effects. In the second step, we used a behavioral genetic approach to assess whether effects detected in our SEM were due to genetic or environmental factors. We used the MPlus 8 software package to test our models and Robust Weighted Least Squares (WLSMV) ${ }^{7}$ as the estimator because our models contained a mix of categorical and continuous dependent variables (Muthén, du Toit, \& Spisic, 1997). We conducted significance testing at the .05 level and interpreted $p$-values associated with unstandardized estimates.

Step 1-SEM. We hypothesized a measurement model (Figure 2) and full SEM (Figure 3) based on the final models reported in Richardson et al. (2018) and the variables available to us in the Add Health. This model was tested against a randomly selected half of the twins (i.e., twin 1 sample). In the hypothesized measurement model, a latent fragmented family structure factor subsumed parental divorce, unrelated male in household, single mother, adverse childhood experiences, father absence, and childhood sexual abuse. We also hypothesized this factor would subsume relatedness of participants to their father figure. The latent socioeconomic status factor subsumed father education, mother education, family income, and neighborhood danger. We hypothesized father absence and childhood sexual abuse would cross-load on this factor in addition to fragmented family structure.

In our hypothesized full SEM (see Figure 3), birthweight, BMI, relatedness to father figure, paternal military service, and fragmented family structure were expected to have direct effects on age at menarche. In addition, father absence was expected to have direct effects on age at menarche and age at sexual debut. Age at menarche was expected to have a direct effect on age at sexual debut. Age at sexual debut was expected to have

\footnotetext{
${ }^{7}$ We planned to use robust maximum likelihood (MLR) as the estimator but we had a mix of continuous and categorical indicators and needed to specify residual covariances between the latter. This can only be accomplished via mixture modeling in the context of MLR and we found this approach much more computationally intensive than WLSMV. We preregistered the decision to use WLSMV if MLR was too computationally intensive.
} 
direct effects on risky sex, lifetime number of sexual partners, and desired number of children. Finally, we hypothesized that father absence and early environmental stress would have indirect effects on number of sexual partners and risky sexual behavior through age at menarche and age at sexual debut.

Step 2-Genetically informative modeling. After testing our hypothesized SEM against the twin 1 data, we addressed the possibility that detected associations were confounded using univariate and multivariate ACE models (Neale \& Cardon, 2013). Univariate ACE models are commonly used to decompose the variance in single phenotype into additive genetic (A), shared environmental (C), and nonshared environmental (E) components. From these models we can infer the relative importance of genetic and environmental factors. Extending upon univariate models, multivariate ACE models can be used to determine the extent to which associations among phenotypes are due to overlapping A, C, or E factors. From these models we can infer the degree to which a phenotypic association is potentially confounded by genetic and shared environmental factors, as well as interpret significant within pair effects $(b \mathrm{E})$ as consistent with the quasi-causal hypothesis (Turkeimer $\&$ Harden, 2014). The latter inference is possible because only nonshared environmental factors (i.e., environmental factors that vary within pairs) can confound $b \mathrm{E}$ (i.e., genetic and shared environmental factors are controlled). For complete details of our univariate and multivariate ACE model specifications, as well as syntax, see Supplementary Materials. Readers may also consult Turkheimer and Harden (2014) for additional discussion of the specification and interpretation of multivariate ACE models.

Model fit. We preregistered the use of a variety of indices in order to obtain a robust assessment of model fit. We considered the substantive meaningfulness of the model and regarded Tucker-Lewis (TLI) and comparative fit (CFI) indices greater than .95 (Byrne, 2001; Hu \& Bentler, 1999), along with root mean square error of approximation values of less than .05 (RMSEA; Browne \& Cudeck, 1993) as evidence of acceptable fit to the data. We also considered significant $\chi^{2}$ likelihood ratio statistics as evidence that the hypothesis of exact fit should be rejected (Bollen, 1989).

\section{Results}

\section{SEM}

Measurement model. The fit of the hypothesized two-factor measurement model was inadequate (e.g., CFI =.91; see Table 2). The correlation between the SES and fragmented family structure factors was quite large 
( $r=-.95, p<.001)$, suggesting the factors were not distinct. We therefore specified a single-factor model, tested it, and found that it fit worse than the two-factor model (e.g., CFI =.88). There were two relatively large modification indices ( $>10)$ suggesting that we should consider freeing a residual covariance between early childhood sexual abuse and adverse childhood experiences, and between single mother and divorce. These made substantive sense given the first pair of variables assessed adverse experiences, and given the second pair assessed similar aspects of family structure. The loading for the father absence index was smaller than $\beta=.20$, so we specified this indicator as a covariate of the environmental stress factor as well as the other exogenous variables. Following these modifications, we tested the resulting model and fit was improved (e.g., CFI =.92; see Table 2). However, the standardized residual covariance between single mother and divorce exceeded one (i.e., there was a Heywood case). Given the large correlation, we combined single mother and divorce variables by summing them. The resulting model converged normally and fit the data well (e.g., CFI = .96; see Table 2); thus, we accepted it as the best reproducer of the observed associations among the variables (see Figure 4).

Full SEM. We proceeded to test a full SEM that included age at menarche, age at sexual debut, and risky behavior. Based on our measurement model findings (see Figure 4), we specified the single early environmental stress factor rather than the hypothesized fragmented family structure and socioeconomic status factors as impacting age at menarche, age at sexual debut, and risky behavior. We also included age in this model as a covariate because Richardson et al. (2018) detected effects of SES that were attributable to age. The remainder of the full SEM we tested was consistent with the preregistered SEM in Figure 3. Fit to the data was marginal (e.g., CFI $=.91$; see Table 2$)$. Two modification indices were relatively large $(>10)$, suggesting we should consider estimating effects of BMI on number of sexual partners and parental number of children on age at sexual debut. These specifications produced a well-fitting model (e.g., CFI $=.95$; see Table 2 ) and thus we proceeded to interpret our structural regression estimates (see Figure 5; for parameter estimates, see Supplementary Materials).

Early environmental stress had a statistically significant small negative effect on age at sexual debut $(b=$ $.22, p<.01, \beta=-.21)$, but not age at menarche $(p=.38)$. Age at sexual debut had significant large and moderate negative effects on number of sexual partners $(b=-.61, p<.001, \beta=-.52)$ and risky sexual behavior $(b=-.29, p<$ $.001, \beta=-.29)$, respectively. Parental number of biological children had a small significant positive effect on age at sexual debut $(b=.15, p<.01, \beta=.20)$ and BMI had a small significant negative effect on number of sexual 
partners $(b=-.61, p<.001, \beta=-.26)$. Only one variable had a significant effect on age at menarche: BMI, which had a small negative effect $(b=-.33, p<.05, \beta=-.15)$. No effects of age were significant $(p s>.07)$. There was a moderate significant positive association between number of sex partners and risky sexual behavior $(r=.40, p<$ .001). Finally, the indirect effect of early environmental stress on number of sexual partners through age at sexual debut was significant $(b=.14, p<.05, \beta=.11)$, but the indirect effect of early stress on risky sexual behavior was $\operatorname{not}(b=.06, p=.06, \beta=.06)$.

\section{Genetically Informative Modeling}

Univariate ACE. Following our testing of the non-genetically informed full SEM (Figure 5), we specified and tested ACE models for the two primary variables of interest in the current research: age at menarche and age at sexual debut. We specified liability models for both variables because they were ordinal and used theta parameterization. All three ACE components were specified as latent variables and the residuals were fixed to zero. For age at menarche, the ACE model fit the data very well (e.g., $\chi^{2}=26.35, p=.66$; see Table 2 ) and the standardized loadings on $A$ and $E$ were $\beta=.68$ and $\beta=.74$, respectively, suggesting about $45 \%$ of the phenotypic variance was attributable to additive genetic factors while about $55 \%$ of the phenotypic variance was due to nonshared environmental factors (for parameter estimates, see Supplementary Materials). The estimate for C was nil $(b=0.00, \beta=0.00, p=1.00)$, suggesting environmental factors that varied between families did not explain significant variance in age at menarche. We found the same result using parameter constraints instead of latent variables.

For age at sexual debut, the ACE model also fit the data very well (e.g., $\chi^{2}=25.54, p=.85$; see Table 2 ) and the standardized loadings on $\mathrm{A}, \mathrm{C}$, and $\mathrm{E}$ were $\beta=.70, \beta=.42, \beta=.58$, respectively. This suggested about $49 \%$ of the phenotypic variance was attributable to additive genetic factors, about $18 \%$ was due to shared environmental factors, and about $33 \%$ of the variance was due to nonshared environmental factors.

Multivariate ACE. Next, we specified multivariate ACE models to examine the extent to which significant effects of variables in our final SEM were due to additive, shared environmental, and nonshared environmental factors. As a reminder, BMI, age at sexual debut, early environmental stress, and number of children had significant effects in the final SEM (see Figure 5). Importantly, we did not include environmental stress and number of children in our multivariate ACE models because if they were included, their effects would 
have only been attributable to C. This is because they only varied between twin pairs but not within; that is, they were shared environmental factors by definition and their variances were direct estimates of $\mathrm{C}$. Therefore, their phenotypic and shared environmental effects were necessarily identical. This does not imply the effect of early environmental stress, for instance, was not confounded by genetic factors that varied between families. However, our multivariate models were not informative regarding this possibility. It is worth noting, however, that we were able to draw inferences about genetic confounding of early environmental stress effects on the basis of the univariate ACE estimates (see Discussion).

We first specified a multivariate ACE model that included BMI and the two endogenous variables to which it was significantly associated: age at menarche and number of sex partners (see Figure 6). In this model, the two endogenous variables were regressed on the BMI ACE components. We tested this model and fit was marginal (e.g., TLI = .94; RMSEA = .06; see Table 2). There was a significant small and negative effect of A on number of sex partners $(\beta=-.28, p<.05$; for parameter estimates, see Supplementary Materials). According to the Wald $z$-tests, no other ACE effects on number of sex partners or age at menarche were significant $(p s>.13)$. We next used the corrected global $\chi^{2}$ test to compare the initial multivariate ACE model to restricted models in which the non-significant $\mathrm{A}, \mathrm{C}$, and E effects on number of sex partners and age at menarche were constrained to zero. ${ }^{8}$ Constraining the effect of $\mathrm{E}$ on number of sex partners to zero did not significantly degrade fit $(\Delta \chi=.48$ [1], $p=.49)$, nor did constraining to zero the effect of E on age at menarche $(\Delta \chi=.18[1], p=.69)$. Constraining to zero the effect of $\mathrm{C}$ on number of sexual partners also did not significantly degrade fit $(\Delta \chi=1.83[1], p=.18)$; however, constraining to zero the effect of $\mathrm{C}$ on age at menarche did worsen fit $(\Delta \chi=5.02[1], p=.03)$. Thus, we interpreted this effect, which was moderate and positive $(\beta=.35)$. Finally, constraining to zero the effect of A on age at menarche significantly degraded fit $(\Delta \chi=7.63[1], p=.01)$, so we also interpreted this (small and negative) $\operatorname{effect}(\beta=-.26)$.

\footnotetext{
${ }^{8}$ The Wald $z$-tests and global $\chi^{2}$ test are only a symptotically equivalent, meaning that they will only match closely in large samples. Wald tests are regarded as a poor choice to testing hypotheses in samples of modest size, where second-order parameters converge slowly to normality. Additionally, the Wald $z$-test is sensitive to the choice of parameterization, whereas the global $\chi^{2}$ test is invariant to change-of-parameter.
} 
Next, we specified a multivariate ACE model for the age at sexual debut effects on risky behavior and number of sexual partners (see Figure 6). We tested the model and fit was excellent (e.g., $\chi^{2}=89.75$ [88], $p=.43$; see Table 2). There were significant large and moderate negative effects of $\mathrm{A}(\beta=-.51, p<.001)$ and $\mathrm{E}(\beta=-.32$, $p<.01)$ on number of sex partners. There was also a large significant negative effect of $A$ on risky behavior $(\beta=-$ $.60, p<.05)$. The effects of $\mathrm{C}$ on number of sex partners and risky behavior were both non-significant $(p s>.17)$, as was the effect of E on risky behavior $(p=.05)$. Constraining the effect of $\mathrm{C}$ on number of sexual partners to zero did not significantly degrade fit $(\Delta \chi=.89[1], p=.35)$, nor did constraining the effect of $E$ on risky behavior to zero $(\Delta \chi=2.08$ [1], $p=.15)$. However, constraining the effect of $\mathrm{C}$ on risky behavior did significantly degrade fit $(\Delta \chi=8.25[1], p=.004)$ and thus we proceeded to interpret this effect, which was moderate and positive $(\beta=$ $.44)$.

\section{Discussion}

The current research tested differential predictions of several life history developmental models for age at menarche and age at sexual debut (see Figure 1), including paternal investment theory (Draper \& Harpending, 1982), psychosocial acceleration theory (Belsky et al., 1991), and child development theory (Ellis, 2004). We preregistered seven specific research aims and a research strategy utilizing a twin subsample of the National Longitudinal Study of Adolescent to Adult Health (Add Health). Strengths of our approach included the use of a subsample drawn from a nationally representative sample, the comprehensive nature of the SEM in step one, and the genetically informative design in step two. Table 3 provides a summary of each specific aim with accompanying results. Overall, our results provide no comprehensive support for any single sexual development model (i.e., paternal investment, psychosocial acceleration, or child development theory), although analyses supported some aspects of each model tested.

Each of the evolutionary life history-derived models of sexual development in girls, discussed here, are underscored by the fundamental prediction that features of the rearing environment predict pubertal timing (Ellis et al., 2004; Ellis et al., 2009; Belsky et al., 2019). These models differ only in why menarche timing is purported to accelerate in stressful environments. Patemal investment theory (Draper \& Harpending, 1982) proposes the special role of the father as a signal of environmental risk; psychosocial acceleration theory (Belsky et al., 1991) highlights the utility of adjusting maturation to facilitate optimal adult reproductive strategies; and child 
development theory (Ellis, 2004) posits optimal regulation of juvenile duration with little predictions regarding adult reproductive strategies. Our SEM results, however, showed no evidence that age at menarche reflected father absence or early environmental stress as predicted by each of the theories (see Figure 5); the only significant predictor of age at menarche in this study was body mass index (BMI). The lack of significant effects of the life history antecedents on menarche timing has important implications for each of these evolutionary life history models of sexual development.

With regard to paternal investment theory, the null association we found between years of father absence and age at menarche joins a growing list of studies that have failed to find a significant effect of father absence in diverse samples (see Kyweluk et al., 2017; Sheppard et al., 2014; Smith, 2017; Sohn, 2017; TenEyck et al., 2018). Collectively, these results suggest that the specific role of the father is likely a spurious, or context-dependent effect. The effect of father absence in earlier studies might be due to omission of potential confounders (Richardson et al., 2018; Webster et al., 2014) or biased sampling given that most studies on father absence are conducted in WEIRD countries. A systematic review by Sear et al. (2019) shows no consistent effect of father absence accelerating menarche, and in a substantial minority of cases father absence appears to have the opposite effect of what paternal investment theory proposes. The mixed results of the impact of fathers on daughters in cross-cultural research accords with abundant documentation that high patemal investment - normative in the West - is not a universal feature of human families (Sear et al., 2008, 2018) and that paternal investment is highly variable across human cultures and history (Quinlan, 2008). Even assuming that menarche timing is plastic in response to early environments, if father absence does have a discernable impact on female development it is plausible that such an effect is limited to cultures with high paternal investment norms. From an evolved cognition approach, this would suggest that the father does not have a "distinctive influence" (see Belsky, 2019) per se, but rather that in specific cultures, father absence tracks broader adult-investment cues that children are sensitive to in early development.

Another explanation for the spurious nature of the father absence effect is the genetic confounding hypothesis (Barbaro et al., 2017; Rowe, 2002). The current study does not provide evidence in support of the genetic confounding hypothesis because there was no documented effect between father absence and menarche to be confounded. Moreover, the non-significant effect of shared environmental factors (i.e., C) suggests that not 
only did father absence and other measured environmental factors play no significant role in menarche timing; no unmeasured factors that varied between families played such a role either. Also consistent with this conclusion are findings from studies using candidate gene (Schlomer et al., 2019) and polygenetic score (Gaydosh et al., 2018) approaches, which have not supported the genetic confounding hypothesis. A more conclusive test of the genetic confounding hypothesis in future research should use a higher-powered twin study or Genome Wide Association Study (GWAS) to provide more accurate genetic correlation estimates between father absence and menarche timing variables, which could then be used to directly test the simulation-model predictions presented by Barbaro et al. (2017).

Our study was unable to explore key tests of paternal investment theory related to the proposed distinctive role of the father by examining genetic relatedness of father-figures in the home and father military service due to lack of variance on these measures in our dataset. To comprehensively examine the role of the father within the context of life history theory developmental models, reasons for father absence beyond active non-investment or abandonment must be investigated. Similarly, the subtle distinction between father absence accelerating menarche and father presence need to more carefully be examined moving forward. Future investigations of paternal investment theory would benefit by engaging with these questions.

With regard to psychosocial acceleration theory in particular, age at menarche is proposed to be key regulatory mechanism that is posited to influence a cascade of processes related to reproductive and sexual strategies (Belsky, 2019). The current study did not document a significant effect of environmental stress, indicated by socioeconomic variables and family structure variables, on menarche timing as the theory would predict. Instead, our model shows a significant effect of environmental stress on age at sexual debut, consistent with recent research (Snopkowski \& Ziker, 2020). Because age at sexual debut can be seen more cognitively and socially mediated, relative to age at menarche, these findings seem consistent with theoretical and empirical work suggesting that harshness and unpredictability impact life history indicators through unpredictability schemas (Cabeza de Baca et al., 2016), future discounting (Hill et al., 2008), present time perspective (Copping et al., 2014; Kruger et al., 2008), and shorter subjective life expectancies (Chisholm et al., 2005).

Although not specified by psychosocial acceleration theory, the notion that early environmental stress is associated with a more unrestricted reproductive strategy, one marked by earlier sexual activity with more sexual 
partners, is broadly in line with the essence of the theory; importantly, however, our results suggest that the regulatory mechanism may be missing. The post-menarche associations proposed by psychosocial acceleration theory are, however, supported by our data such that earlier sexual debut is significantly associated with subsequent risky sexual behavior and a greater number of sexual partners - outcomes consistent with a fast life history strategy or unrestricted sociosexual orientation.

Finally, the results of our study show some consistency with the predictions of child development theory (Ellis, 2004). But, in direct contrast to theoretical predictions, age at menarche was not associated with any measured features of the early environment tested in this study, nor was age at menarche predictive of age at sexual debut. In addition, as mentioned above, sexual debut was predictive of reproductive strategy, associations about which child development theory is relatively agnostic (Ellis, 2004). If, however, child development theory operationalizes the end of childhood as engagement in sexual activity rather than strictly sexual maturation, then conceivably the results presented here could accord with the essence of the theory, as suggested for psychosocial acceleration theory above.

The results of our behavior genetic models do provide some support for a broader underlying assumption of life history developmental models of sexual development: reproductive strategies may be adjusted in response to early environmental conditions. One approach to evaluating this foundational assumption is to examine the relative importance of genetic, shared environmental, and nonshared environmental sources of variance in indicators of reproductive strategies, as we did in the current study. Proponents of the facultative adjustment assumption would argue that shared environmental variation (often conceptualized as the rearing and home environment; but, more accurately, any environmental variation shared by two individuals that make them more phenotypically similar) explains a proportion the variance in such indicators. The genetic confounding hypothesis (Barbaro et al., 2017; Rowe, 2002) proposes, rather, that the phenotypic variance in these indicators is explained predominantly by genetic variation underlying the traits of interest.

Consistent with facultative adjustment, our univariate ACE model estimates are consistent with important roles of both shared environmental (e.g., early environmental stress) and shared genetic factors in the etiology of age at sexual debut. That is, both genetic and environmental factors that vary between twin pairs appear to account for the variance in this phenotype. The rest of the phenotypic variance is attributable to nonshared 
environmental factors. Contrary to facultative adjustment, however, only genetic and nonshared environmental factors explained significant variance in age at menarche, suggesting (as mentioned earlier) that early environmental stress and its indicators, as well as any other pairwise variables such as father absence, do not appear to play an important role in the etiology of menarche timing. These findings are consistent with the confounding position (see Barbaro et al., 2017).

Multivariate ACE models fit to age at sexual debut, number of sexual partners, and risky behavior (see Figure 6) suggest that the association between age at sexual debut and number of sexual partners was partly confounded by genetic factors, was not due to environmental factors that vary between families, and was partly due to a moderate negative within pair effect (i.e., consistent with the quasi-causal hypothesis). Taken together, these findings are consistent with the notion that although age at sexual debut covaried with number of sexual partners partly because of common genetic factors, earlier debut also caused increased numbers of partners, perhaps by simply increasing time window in which females were sexually active. Results also suggest the association between age at sexual debut and risky behavior w as confounded by genetic and shared environmental factors; the lack of an E effect is inconsistent with the hypothesis that the sexual debut caused risky behavior. Interestingly, the effects of $\mathrm{A}$ and $\mathrm{C}$ on risky behavior were opposite in sign, suggesting ge netic factors that tended to increase age at sexual debut also decreased liability to risky behavior, whereas shared environmental factors that increased age at sexual debut tended to increase risky behavior.

Multivariate ACE models also suggested the association between BMI and number of sexual partners (see Figure 6) was confounded by genetic factors, whereas the association between BMI and age at menarche was due to both genetic and shared environmental factors. Genetic factors that ten ded to decrease BMI also appeared to increase number of sexual partners. Interestingly, A and C effects on age at menarche were opposite in sign, suggesting that genetic factors that worked to increase BMI also decreased age at menarche, whereas shared environmental factors that increased BMI also increased age at menarche. The lack of E effects is inconsistent with the quasi-causal hypothesis that BMI caused either outcome.

\section{Conclusion}

Life history models of pubertal development and reproductive strategies have been instrumental in understanding menarche timing in girls from an evolutionary perspective. The current study evaluated a variety of 
predictions from prominent life history models - paternal investment theory (Draper \& Harpending, 1982), psychosocial acceleration theory (Belsky et al., 1991), and child development theory (Ellis, 2004) - with the broader aims of: (1) comprehensively evaluating life history antecedents of menarche timing and sexual debut, and (2) extending our understanding of these life history models within the context of a genetically informative design. The results of this research show mixed support for varied aspects of each theoretical model, and this study did not provide evidence for effects of any of the measured life history antecedents (in this study) on age at menarche (see Figure 5). Moreover, the lack of a shared environmental effect on age at menarche suggests that unmeasured environmental factors that vary between families are unlikely to play an important role. These findings pose a problem for each life history model because each specifies menarche timing as a key regulatory mechanism that is responsive to early environmental features. Findings associated with age at sexual debut are more consistent with the models we tested in that variance in this phenotype reflected shared environmental factors and was associated as predicted with early environmental features. Collectively, the results of this research call to question the underlying assumptions of life history derived models of sexual development particularly with regard to menarche timing as a regulatory mechanism, and also highlights the need for genetically informative research in this area to parse out the roles of environmental and genetic factors in these proposed developmental processes. 


\section{References}

Agrawal, A. A., Conner, J. K., \& Rasmann, S. (2010). Tradeoffs and negative correlations in evolutionary ecology. In M.A. Bell, D.J. Futuyma, W.F. Eanes \& J.S. Levinton (Eds.), Evolution after Darwin: The First 150 Years (pp. 243-268). Sinauer.

Anderson, K. G. (2015). Father absence, childhood stress, and reproductive maturation in South Africa. Human Nature, 26, $401-425$.

Barbaro, N., Boutwell, B. B., Barnes, J. C., \& Shackelford, T. K. (2017). Genetic confounding of the relationship between father absence and age at menarche. Evolution and Human Behavior, 38, 357-365.

Barnes, J. C., \& Boutwell, B. B. (2013). A demonstration of the generalizability of twin -based research on antisocial behavior. Behavior Genetics, 43, 120-131.

Barnes, J. C., Boutwell, B. B., Beaver, K. M., Gibson, C. L., \& Wright, J. P. (2014). On the consequences of ignoring genetic influences in criminological research. Journal of Criminal Justice, 42, 471-482.

Belsky, J. (2019). Early-life adversity accelerates child and adolescent development. Current Directions in Psychological Science, 28, 241-246.

Belsky, J., Schlomer, G. L., \& Ellis, B. J. (2012). Beyond cumulative risk: Distinguishing harshness and unpredictability as determinants of parenting and early life history strategy. Developmental Psychology, 48, 662-673.

Belsky, J., Steinberg, L., \& Draper, P. (1991). Childhood experience, interpersonal development, and reproductive strategy: An evolutionary theory of socia lization. Child Development, 62, 647-670.

Belsky, J., Steinberg, L., Houts, R. M., \& Halpern-Felsher, B. L. (2010). The development of reproductive strategy in females: Early maternalharshness $\rightarrow$ earlier menarche $\rightarrow$ increased sexualrisk taking. Developmental Psychology, $46,120-128$.

Bjorklund, D. F. (2016). Incorporating development into evolutionary psychology: Evolved probabilistic cognitive mechanisms. Evolutionary Psychology, 14 .

Blouin, S. F. \& Blouin, M. (1988). Inbreeding avoidance behaviors. Trends Ecology and Evolution, 3, $230-233$.

Bollen, K. A. (1989). A new incrementalfit index for general structuralequation models. Sociological Methods \& Research, $17,303-316$.

Boynton-Jarrett, R., Wright, R. J., Putnam,F. W., Hibert, E. L., Michels, K. B., Forman, M. R., \& Rich-Edwards, J. (2013). Childhood abuse and age at menarche. Journal of Adolescent Health, 52, 241-247. 
Braendle, C., Heyland,F., \& Flatt, T. (2011). Integrating mechanistic and evolutionary analysis of life history variation. In T. Flatt \& F. Heyland (Eds.), Mechanisms of life history evolution. The genetics and physiology of life history traits and trade-offs (pp. 3-10). Oxford University Press.

Browne, M. W., \& Cudeck, R. (1993). Alternative ways of assessing model fit. In Bollen, K.A., Long, J.S. (Eds.), Testing structural equation models (pp. 111-135). Sage.

Byrne, B. M. (2001). Structural equation modeling with AMOS, EQS, and LISREL: Comparative approaches to testing for the factorialvalidity of a measuring instrument. International Journal of Testing, 1, 55-86.

Cabeza de Baca,T., Barnett, M. A., \& Ellis, B. J. (2016). The development of the child unpredictability schema: Regulation through maternallife history trade-offs. Evolutionary Behavioral Sciences, 10, 43-55.

Charpentier, M. J., Van Horn, R. C., Altmann, J., \& Alberts, S. C. (2008). Paternaleffects on offspring fitness in a multimale primate society. Proceedings of the National Academy of Sciences, 105, 1988-1992.

Chisholm, J. S., Ellison, P. T., Evans, J., Lee, P. C., Lieberman,L. S., Pavlik, Z., ... Worthman, C M (1993). Death, hope, and sex: Life-history theory and the development of reproductive strategies. Current Anthropology, 34, 1-24.

Chisholm, J. S., Quinlivan, J. A., Petersen, R. W., \& Coall, D. A. (2005). Early stress predicts age at menarche and first birth, adult attachment, and expected lifespan. Human Nature, 16, 233-265.

Copeland, W., Shanahan, L., Miller, S., Costello, E. J., Angold, A., \& Maughan, B. (2010). Outcomes of early pubertal timing in young women: a prospective population-based study. American Journal of Psychiatry, 167, 1218-1225.

Copping, L. T., Campbell, A., \& Muncer, S. (2014). Conceptualizing time preference: A life-history analysis. Evolutionary Psychology, 12(4), 147470491401200411.

Davis, E. C., \& Friel, L. V. (2001). Adolescent sexuality: Disentangling the effects of family structure and family context. Journal of Marriage and Family, 63, 669-681.

Del Giudice, M. (2009). Sex, attachment, and the development of reproductive strategies. Behavioral and Brain Sciences, 32, $1-67$.

Del Giudice, M. (2015). Plasticity as a developing trait: exploring the implications. Frontiers in Zoology, 12, S4.

Del Giudice, M., Gangestad, S. W., \& Kaplan,H. S. (2015). Life history theory and evolutionary psychology. In D. M. Buss (Ed.), The handbook of Evolutionary Psychology (pp. 88-114). Wiley.

D'Onofrio, B. M., Turkheimer, E., Emery, R. E., Slutske, W. S., Heath, A. C., Madden, P. A., \& Martin, N. G. (2006). A genetically informed study of the processes underlying the association between parental maritalinstability and offspring adjustment. Developmental Psychology, 42, 486-499. 
Draper, P., \& Harpending, H. (1982). Father absence and reproductive strategy: An evolutionary perspective. Journal of Anthropological Research, 38, 255-273.

Ellis, B. J. (2004). Timing of pubertalmaturation in girls: An integrated life history approach. Psychological Bulletin, 130, 920-958.

Ellis, B. J., Figueredo, A. J., Brumbach, B. H., \& Schlomer, G. L. (2009). Fundamentaldimensions of environmental risk: The impact of harsh versus unpredictable environments on the evolution and development of life history strategies. Human Nature, 20, 204-268.

Ellis, B. J., Schlomer, G. L., Tilley, E. H., \& Butler, E. A. (2012). Impact of fathers on risky sexual behavior in daugh ters: A genetically and environmentally controlled sibling study. Development and Psychopathology,24,317-332.

Figueredo, A. J., Vásquez, G., Brumbach, B. H., \& Schneider, S. (2004). The heritability of life history strategy: The Kfactor, covita lity, and personality. Social Biology, 51, 121-143.

Figueredo, A. J., Vásquez, G., Brumbach, B. H., Schneider, S. M., Sefcek, J. A., Tal, I. R., ... \& Jacobs, W. J. (2006). Consilience and life history theory: From genes to brain to reproductive strategy. Developmental Review, 26, 243275.

Gaydosh,L., Belsky, D. W., Domingue, B. W., Boardman, J.D., \& Harris, K. M. (2018). Father absence and accelerated reproductive development in non-Hispanic white women in the United States. Demography, 55, 1245-1267.

Harris, K. M., Halpern, C. T., Smolen, A., \& Haberstick, B. C. (2006). The nationallongitudinal study of adolescent health (Add Health) twin data. Twin Research and Human Genetics, 9, 988-997.

Hill, E. M., Jenkins, J., \& Farmer, L. (2008). Family unpredictability, future discounting, and risk taking. The Journal of Socio-Economics, 37, 1381-1396.

Hill, K., \& Kaplan,H. (1999). Life history traits in humans: Theory and empirical studies. Annual Review of Anthropology, $28,397-430$.

Hu, L. T., \& Bentler, P. M. (1999). Cutoff criteria for fit indexes in covariance structure analysis: Conventionalcrit eria versus new alternatives. Structural Equation Modeling: A multidisciplinary Journal, 6, 1-55.

Ibitoye, M., Choi, C., Tai, H., Lee, G., \& Sommer, M. (2017). Early menarche: A systematic review of its effect on sexual and reproductive health in low-and middle-income countries. PloS one, 12, e0178884.

Jacobson, K. C., \& Rowe, D. C. (1998). Genetic and shared environmental influences on adolescent BMI: interactions with race and sex. Behavior Genetics, 28, 265-278. 
James, J., Ellis, B. J., Schlomer, G. L., \& Garber, J. (2012). Sex-specific pathways to early puberty, sexual debut, and sexual risk taking: Tests of an integrated evolutionary-developmentalmodel. Developmental Psychology, 48, 687-702

Johnson, W., Turkheimer, E., Gottesman, I. I., \& Bouchard Jr, T. J. (2009). Beyond heritability: Twin studies in behavioral research. Current Directions in Psychological Science, 18, 217-220.

Kaplan,H. S., \& Gangestad, S. W. (2005). Life history theory and evolutionary psychology. In D. M. Buss (Ed.), The Handbook of Evolutionary Psychology (pp.68-95). Wiley.

Kendler, K. S., \& Baker, J. H. (2007). Genetic influences on measures of the environment: A systematic review. Psychological Medicine, 37,615-626.

Kruger, D. J., Reischl, T., \& Zimmerman, M. A. (2008). Time perspective as a mechanism forfunctionaldevelopmental a daptation. Journal of Social, Evolutionary, and Cultural Psychology, 2, 1-22.

Kuzawa,C. W., \& Bragg, J. M. (2012). Plasticity in human life history strategy: Implications for contemporary human variation and the evolution of genus Homo. Current Anthropology, 53, S369-S382.

Kyweluk, M. A., Georgiev, A. V., Borja, J. B., Gettler, L. T., \& Kuzawa, C. W. (2018). Menarcheal timing is accelerated by favorable nutrition but unrelated to developmentalcues of morta lity or familial instability in Cebu, Philippines. Evolution and Human Behavior, 39, 76-81.

La Guardia, A. C., Nelson, J. A., \& Lertora, I. M. (2014). The impact of father absence on daughter sexualdevelopment and behaviors: Implications for professional counselors. The Family Journal, 22, 339-346.

Matchock, R. L., \& Susman, E. J. (2006). Family composition and menarchealage: Anti-inbreeding strategies. American Journal of Human Biology, 18, 481-491.

McGue, M., Osler, M., \& Christensen, K. (2010). Causalinference and observationalresearch: The utility of twins. Perspectives on Psychological Science, 5, 546-556.

Mendle, J., Turkheimer, E., D'Onofrio, B. M., Lynch, S. K., Emery, R. E., Slutske, W. S., \& Martin, N. G. (2006). Family structure and age at menarche: a children-of-twins approach. Developmental Psychology, 42, 533.

Nedelec, J. L., Richardson, G., \& Silver, I. A. (2017). Religiousness, spirituality, and substance use: A genetically sensitive examination and critique. Journal of Drug Issues, 47, 340-355.

Parent, A. S., Teilmann, G., Juul, A., Skakkebaek, N. E., Toppari, J., \& Bourguignon, J. P. (2003). The timing of normal puberty and the age limits of sexual precocity: variations around the world, secular trends, and changes after migration. Endocrine Reviews, 24, 668-693. 
Placek, C. D., \& Quinlan, R. J. (2012). Adolescent fertility and risky environments: a population-level perspective across the lifespan. Proceedings of the Royal Society B: Biological Sciences, 279, 4003-4008.

Polderman, T. J. C., Benyamin, B., de Leeuw, C. A., Sullivan, P. F., van Bochoven, A., Visscher, P. M., \& Posthuma, D. (2015). Meta-analysis of the heritability of human traits based on fifty years of twin studies. Nature Genetics, 47, 702-709.

Promislow, D. E., \& Harvey,P. H. (1990). Living fast and dying young: a comparative analysis of life-history variation a mong mammals. Journal of Zoology, 220, 417-437.

Pusey A, WolfM (1996) Inbreeding a voidance in animals. Trends Ecology Evolution, 11, 201-206.

Quinlan, R. J. (2008). Human pair-bonds: Evolutionary functions, ecological variation, and a daptive development. Evolutionary Anthropology, 17,227-238

Quinlan, R. J. (2010). Extrinsic mortality effects on reproductive strategies in a Caribbean community. Human Nature, 21, 124-139.

Richardson, G. B., Dariotis, J. K., \& Lai, M. H. (2017a). From environment to mating competition and Super-K in a predominantly urban sample of young a dults. Evolutionary Psychology, 15.

Richardson, G. B., La Guardia, A. C., \& Klay, P. M. (2018). Determining the roles of father absence and age at menarche in female psychosocialacceleration. Evolution and Human Behavior, 39, 437-446.

Richardson, G. B., Sanning, B. K., Lai, M. H., Copping, L. T., Hardesty, P. H., \& Kruger, D. J. (2017b). On the psychometric study of human life history strategies: State of the science and evidence of two independent dimensions. Evolutionary Psychology, 15.

Romans, S. E., Martin, J. M., Gendall, K., \& Herbison, G. P. (2003). Age of menarche: The role of some psychosocial factors. Psychological Medicine, 33,933-939.

Rowe, D. C. (2002). On genetic variation in menarche and age at first sexual intercourse: A critique of the Belsky-Draper hypothesis. Evolution and Human Behavior, 23, 365-372.

Schlomer, G. L., Murray, J., Yates, B., Hair, K., \& Vandenbergh, D. J. (2019). Father absence, age at menarche, and sexual behaviors in women: Evaluating the genetic confounding hypothesis using the androgen receptor gene. Evolutionary Behavioral Sciences, 3, 205-222.

Sear, R., Sheppard,P., \& Coall, D. A. (2019). Cross-cultural evidence does not support universal acceleration of puberty in father-absent households. Philosophical Transactions of the Royal Society B, 374, 20180124.

Shenk, M. K., Starkweather, K., Kress, H. C., \& Alam, N. (2013). Does absence matter? Human Nature, 24, 76-110. 
Sheppard,P., Snopkowski, K., \& Sear, R. (2014). Father absence and reproduction-related outcomes in Malaysia, a transitionalfertility population. Human Nature, 25, 213-234.

Simpson, J. A., Griskevicius, V., \& Kim, J. S. (2011). Evolution, life history theory, and personality. In L. M. Horowitz \& S. Strack (Eds.), Handbook of interpersonal psychology: Theory, research, assessment, and therapeutic interventions (pp. 75-89). Wiley.

Simpson, J. A., Griskevicius, V., Kou, S. I.-C., Sung, S., \& Collins, A. (2012). Evolution, stress, and sensitive periods: The influence of unpredictability in early versus late childhood on sex and risky behavior. Developmental Psychology, 48, 674-686.

Smith, D. (2017). O brother, where art thou? Investment in siblings for inclusive fitness benefits, not father absence, predicts earlier age at menarche. Biologyletters, 13, 20170464.

Snopkowski, K., \& Ziker, J. P. (2020). Sexualinitiation among Canadian youth: A modelcomparison approach of evolutionary hypotheses shows greatest support for extrinsic mortality cues, intergenerational conflict, and early life psychosocialstressors. Evolution and Human Behavior, 41, 105-116.

Sohn, K. (2017). The null relation between father absence and earlier menarche. Human Nature, 28, 407-422.

Stearns, S. (1992). The evolution oflife histories. Oxford University Press.

Stearns, S. C. (1976). Life-history tactics: a review of the ideas. The Quarterly Review of Biology, 51, 3-47.

TenEyck, M. F., El Sayed, S. A., \& Barnes, J. C. (2019). The effect of absent biological father on female biological maturity: Results from a nationally representative sample of adolescents. Journal of Contemporary Criminal Justice, 35, 3651.

Tither, J. M., \& Ellis, B. J. (2008). Impact of fathers on daughters' a ge at menarche: A genetically- and environmentallycontrolled sibling study. Developmental Psychology, 44, 1409-1420.

Turkheimer, E., \& Harden, K. P. (2014). Behavior genetic research methods: Testing quasi-causalhypotheses using multivariate twin data.In H.T. Reis \& C. M. Judd (Eds.), Handbook of research methods in social and personality psychology (p. 159-187). Cambridge University Press.

Webster, G. D., Graber, J. A., Gesselman, A. N., Crosier, B. S., \& Schember, T. O. (2013). A life history theory of father absence and menarche: A meta-analysis. Evolutionary Psychology, 12, 273-294.

Wright, J. P., Barnes, J. C., Boutwell, B. B., Schwartz, J. A., Connolly, E. J., Nedelec, J. L., \& Beaver, K. M. (2015). Mathematical proof is not minutiae and irreducible complexity is not a theory: A final response to Burt and Simons and a call to criminologists. Criminology, 53, 113. 
Table 1. Descriptive Statistics

\begin{tabular}{|c|c|c|c|c|c|c|c|c|}
\hline & $\begin{array}{c}\text { Twin } \\
\#\end{array}$ & $\begin{array}{l}\text { Add Health } \\
\text { Label }\end{array}$ & Coding & Wave & Min & $\operatorname{Max}$ & $\overline{\mathrm{x}}$ & $\sigma$ \\
\hline Age & 1 & S1 & N/A & $\mathrm{SCH}$ & 13 & 20 & 16.00 & 1.58 \\
\hline Birthweight & 1 & PC19A_P & N/A & 1 & 3 & 9 & 5.02 & 1.18 \\
\hline BMI & 1 & N/A & N/A & 1 & 14.17 & 48.06 & 22.06 & 4.62 \\
\hline \multirow{2}{*}{ Age at menarche } & 1 & H3DA45 & N/A & 3 & 9 & 22 & 12.71 & 1.64 \\
\hline & 2 & H3DA45 & N/A & 3 & 9 & 22 & 12.91 & 1.93 \\
\hline \multirow[t]{2}{*}{ Age at sexualdebut } & 1 & H4SE7 & N/A & 4 & 9 & 27 & 17.20 & 2.84 \\
\hline & 2 & H4SE7 & N/A & 4 & 12 & 26 & 17.20 & 2.63 \\
\hline \multirow[t]{2}{*}{ \# vaginal sex partners } & 1 & H4SE8 & N/A & 4 & 0 & 100 & 7.88 & 10.70 \\
\hline & 2 & H4SE8 & N/A & 4 & 0 & 300 & 7.69 & 21.12 \\
\hline Early sexual abuse & 1 & N/A & Index & N/A & 0 & 1 & 0.08 & 0.28 \\
\hline $\begin{array}{l}\text { Adverse childhood } \\
\text { experiences }\end{array}$ & 1 & N/A & Index & N/A & 0 & 7 & .79 & 1.50 \\
\hline \multirow{2}{*}{ Risky behavior } & 1 & N/A & Index & N/A & -1.02 & 6.17 & 0.00 & 1.00 \\
\hline & 2 & N/A & Index & N/A & -0.97 & 5.89 & 0.00 & 1.00 \\
\hline Parent income & N/A & PA55 & Thousands & PQ & 0 & 800 & 50.37 & 66.58 \\
\hline Parent education & N/A & & $\begin{array}{l}1=8^{\text {th }} \text { grade or less } \\
2=>8^{\text {th }} \text { grade but didn't } \\
\text { graduate } \\
3=\text { business/ trade/ } \\
\text { voc/HS/GED } \\
4=\text { business/ trade/ voc post } \\
\text { HS/ college but non-grad } \\
5=\text { college grad } \\
6=\text { prof training post college }\end{array}$ & PQ & 1 & 6 & 3.60 & 1.35 \\
\hline Mother PAS & 1 & H1RM9 & $\begin{array}{l}0=\text { No } \\
1=\text { Yes }\end{array}$ & 1 & 0 & 1 & 0.13 & 0.34 \\
\hline Neighborhood quality & & N/A & Index & N/A & -1.88 & 1.32 & 0.00 & 1.00 \\
\hline Fatherabsence & 1 & N/A & Index & N/A & -0.46 & 4.27 & 0.00 & 1.00 \\
\hline Divorce/separated & N/A & $\begin{array}{l}\text { Dummy } \\
\text { variable from } \\
\text { PA10 }\end{array}$ & $\begin{array}{l}0=\text { Not divorced } / \text { separated } \\
1=\text { Divorced } / \text { separated }\end{array}$ & PQ & 0 & 1 & 0.15 & 0.36 \\
\hline Single mother & N/A & $\begin{array}{l}\text { Dummy } \\
\text { variable from } \\
\text { PA10 }\end{array}$ & $\begin{array}{l}0=\text { Not single } \\
1=\text { Single }\end{array}$ & PQ & 0 & 1 & 0.07 & 0.26 \\
\hline Married & N/A & $\begin{array}{l}\text { Dummy } \\
\text { variable from } \\
\text { PA10 }\end{array}$ & $\begin{array}{l}0=\text { Not married } \\
1=\text { Married }\end{array}$ & PQ & 0 & 1 & 0.57 & 0.50 \\
\hline \# children bio parents & 1 & H1HR14 & N/A & 1 & 2 & 14 & 3.27 & 1.46 \\
\hline
\end{tabular}


Table 2. Summary of model fit indices.

\begin{tabular}{|c|c|c|c|c|c|c|}
\hline Model & $\chi^{2}$ & $d f$ & $p$ & CFI & TLI & RMSEA \\
\hline \multicolumn{7}{|l|}{ Measurement Models (MM) } \\
\hline 2-Factor Measurement Model* & 95.81 & 48 & $<.001$ & .91 & .88 & $.06(.04-.08)$ \\
\hline 1-Factor MM 1 & 114.50 & 51 & $<.001$ & .88 & .84 & $.07(.05-.09)$ \\
\hline 1 Factor MM2 & 86.90 & 46 & $<.001$ & .89 & .89 & $.06(.04-.07)$ \\
\hline 1 Factor MM3 & 61.68 & 37 & .01 & .94 & .94 & $.05(.03-.07)$ \\
\hline \multicolumn{7}{|c|}{ Structural Equation Models (SEM) } \\
\hline Full SEM1 & 142.51 & 88 & $<.001$ & .91 & .88 & $.05(.03-.06)$ \\
\hline Full SEM2 & 120.21 & 86 & .01 & .95 & .92 & $.04(.02-.06)$ \\
\hline \multicolumn{7}{|l|}{ Univariate ACE Models } \\
\hline Age at Menarche & 26.35 & 30 & .66 & 1.00 & 1.00 & $.00(.00-.06)$ \\
\hline Age at Sexual Debut & 25.54 & 34 & .85 & 1.00 & 1.00 & $.00(.00-.04)$ \\
\hline \multicolumn{7}{|l|}{ Multivariate ACE Models } \\
\hline BMI & 111.27 & 75 & .004 & .85 & .94 & $.06(.04-.08)$ \\
\hline Age at Sexual Debut & 89.75 & 88 & .43 & 1.00 & 1.00 & $.01(.00-.05)$ \\
\hline
\end{tabular}

Notes: *preregistered measurement model; best fitting model in bold. 
Table 3. Summary of research aims and results.

\begin{tabular}{ll}
\hline \hline Research Aim & Results Summary \\
\hline $\begin{array}{l}\text { 1. Assess the association between } \\
\text { father absence and age at menarche }\end{array}$ & No association found between father absence and age at \\
while controlling forfamilial structure & \\
and socioeconomic status &
\end{tabular}

\begin{tabular}{l}
\hline . Assess the association between $\quad$ Did not assess - insufficient data \\
father military service and age at \\
menarche
\end{tabular}

3. Assess the association between Did not assess - insufficient data
father-figure genetic relatedness and
age at menarche

\begin{tabular}{ll}
\hline $\begin{array}{l}\text { 4. Assess the direct association } \\
\text { between birthweight and age at }\end{array}$ & No direct association between birthweight and age at \\
menarche, and the indirect association & menarche, and therefore, no indirect association via BMI. \\
via body mass index (BMI) & $\begin{array}{l}\text { Direct, negative association found between BMI and age at } \\
\text { menarche. }\end{array}$
\end{tabular}
5. Assess direct association between family structure and sexual debut, and the indirect association via age at menarche
(1) Direct association found between latent early environmental stress factor (which includes indicators of family structure) and earlier sexual debut.
(2) No indirect association via age at menarche.
(3) Latent early environmental stress factor did not predict age at menarche.
(4) Age at menarche did not predict age at sexual debut.
6. Assess associations between sexual Direct, negative associations found between earlier sexual debut and reproductive outcomes debut and (1) number of sexual partners, and (2) risky sexual behavior.
7. Assess the robustness of the comprehensive structural equation model (SEM) to genetic controls

(1) Association between sexual debut and number of partners partly confounded by genetic factors and not confounded by shared environmental factors. Nonshared environmental covariance consistent with causal effect of sexual debut on number of number of partners (i.e., quasi-causal hypothesis). (2) Association between sexual debut and risky sexual behavior confounded by genetic factors and shared environmental factors, suggesting no causal effect of sexual debut on risky sexual behavior.

(3) Association between BMI and number of partners confounded by genetic factors.

(4) Association between BMI and age at menarche confounded by genetic and shared environmental factors, suggesting no causal effect of BMI on age at menarche. 


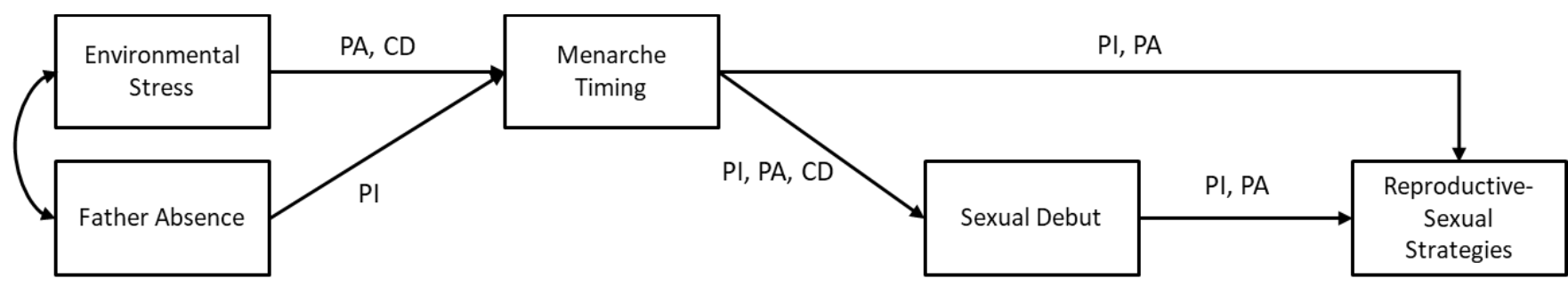

Age

Figure 1. Conceptual model of the theoretical predictions derived from various life history models of development. Solid lines indicate predicted association. PA = psychosocial acceleration theory; $C D=$ child development theory; PI = paternal investment theory. Not pictured are predictions about genetic confounding hypotheses, which are broadly tested to discern the extent to which genetic variation can explain any of the above predicted associations. 


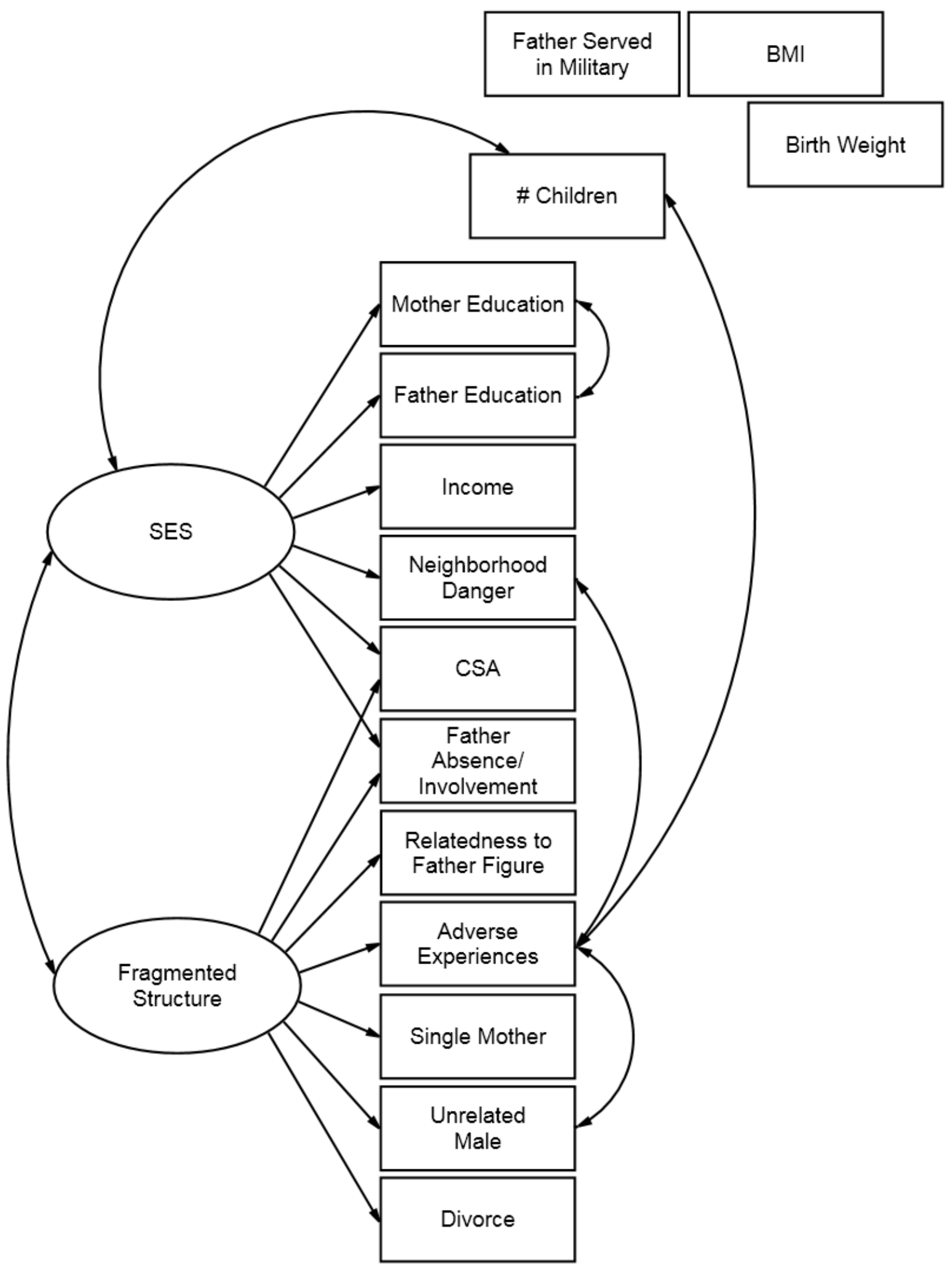

Figure 2. Hypothesized, pre-registered measurement model of early environmental stress. 


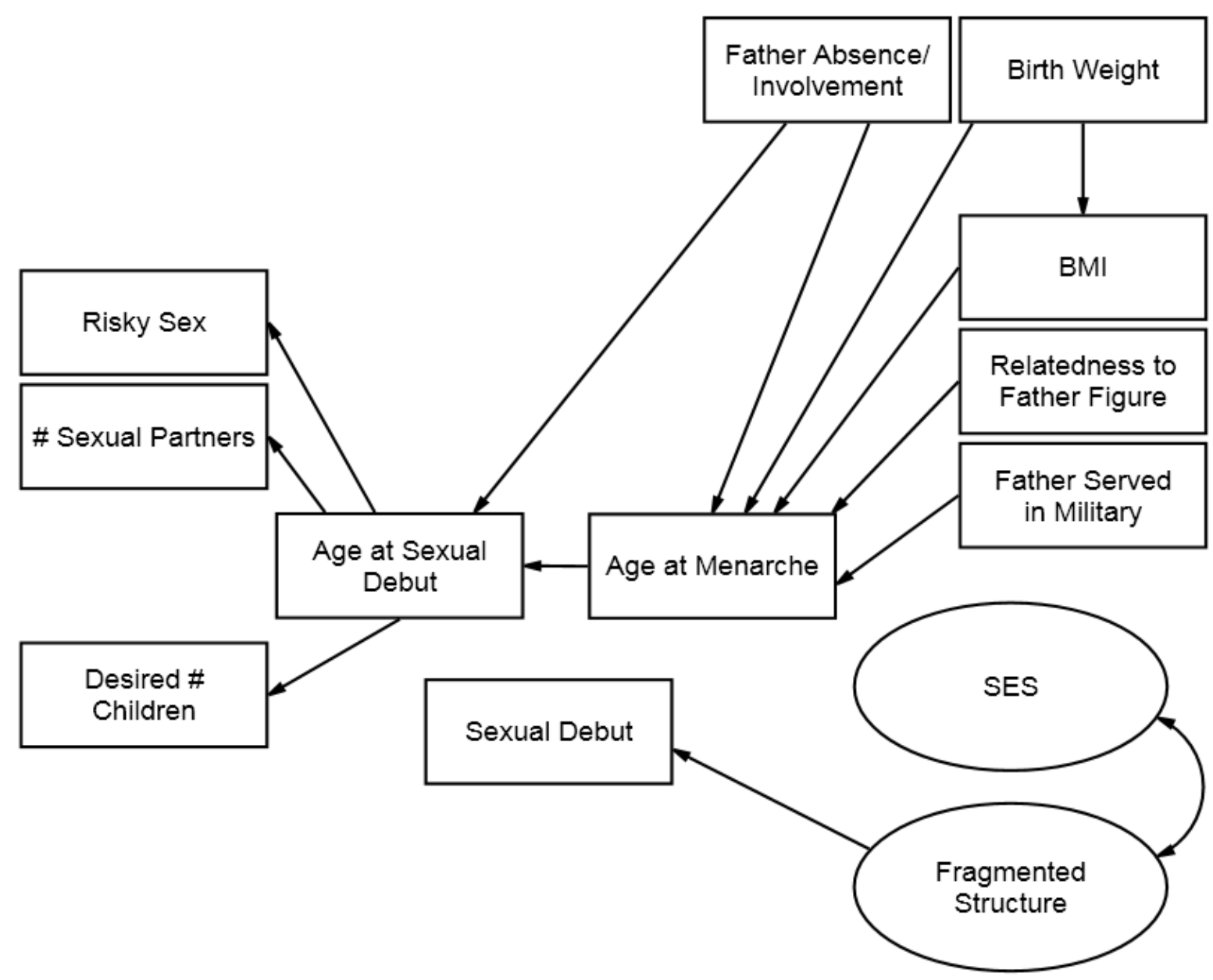

Figure 3. Hypothesized, pre-registered full SEM model of age at menarche and sexual debut, based on Richardson et al. (2018). 


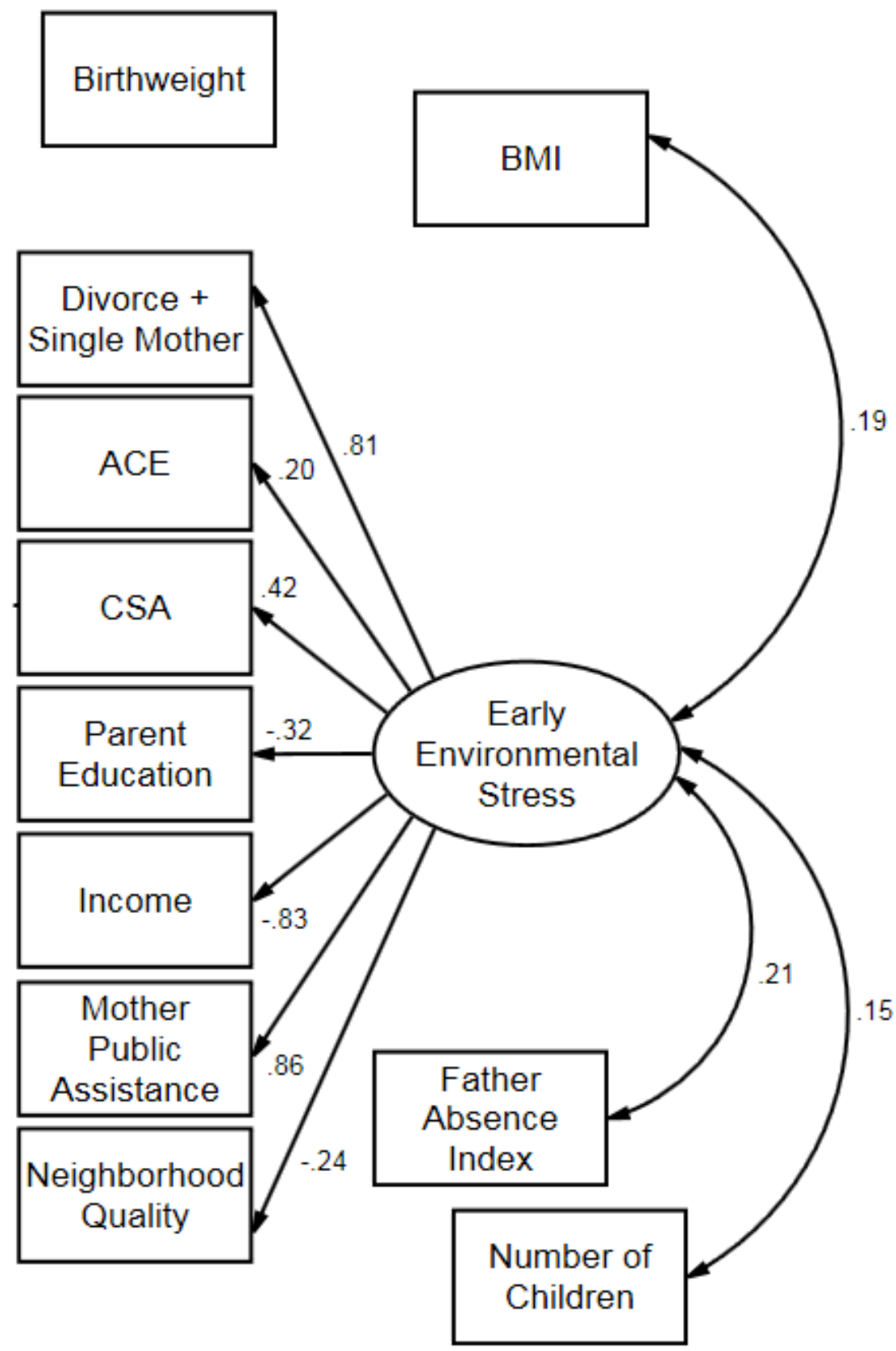

Figure 4. Final measurement model (1 Factor MM3) of life history antecedents. Only significant effects shown. 


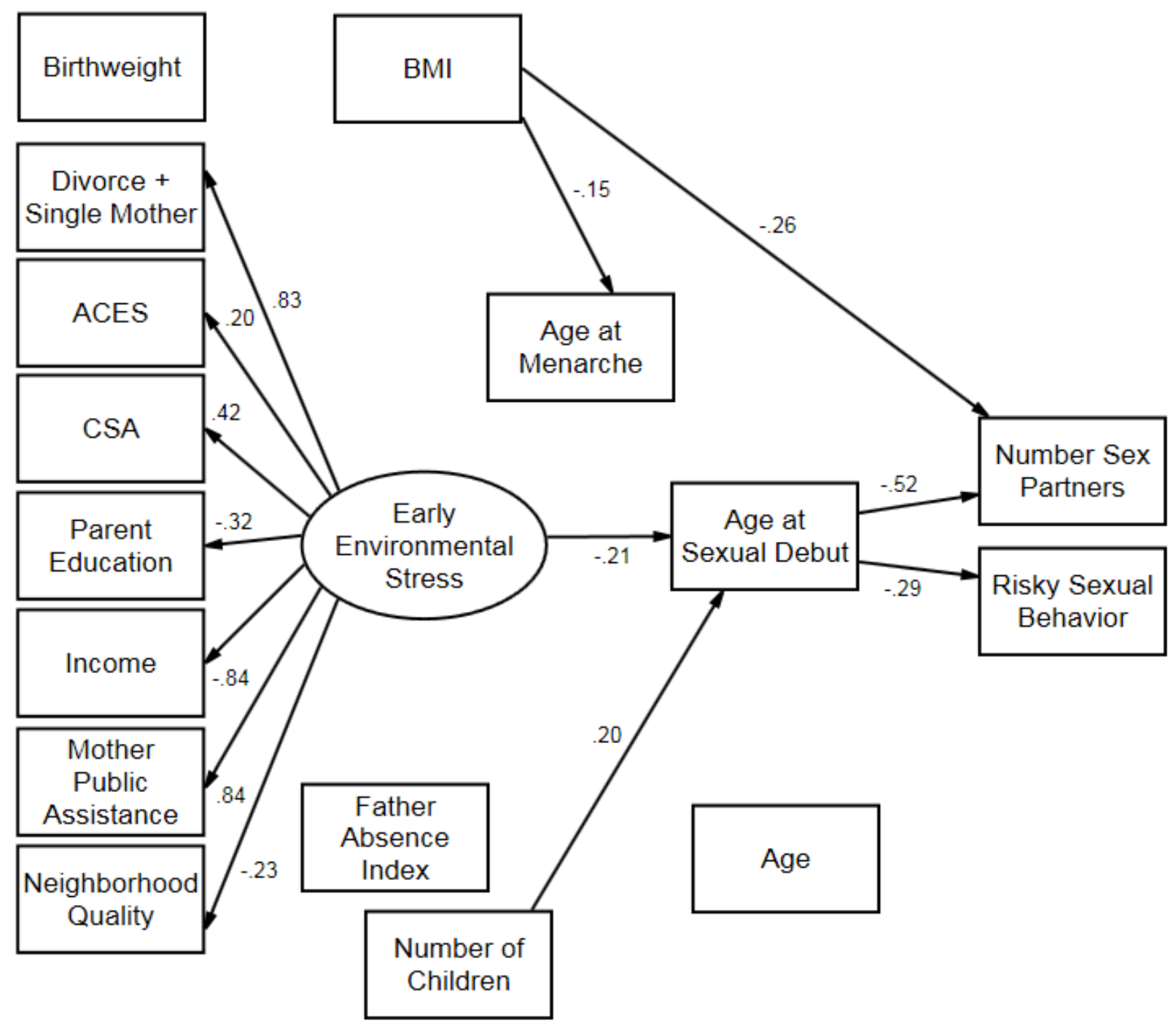

Figure 5. Full SEM2 model. Only statistically significant effects (standardized) displayed. Covariances and residuals omitted for conceptual clarity. $\mathrm{ACES}=$ Adverse childhood experiences. $\mathrm{CSA}=\mathrm{Childhood}$ sexual abuse. $\mathrm{BMI}=$ Body mass index. 


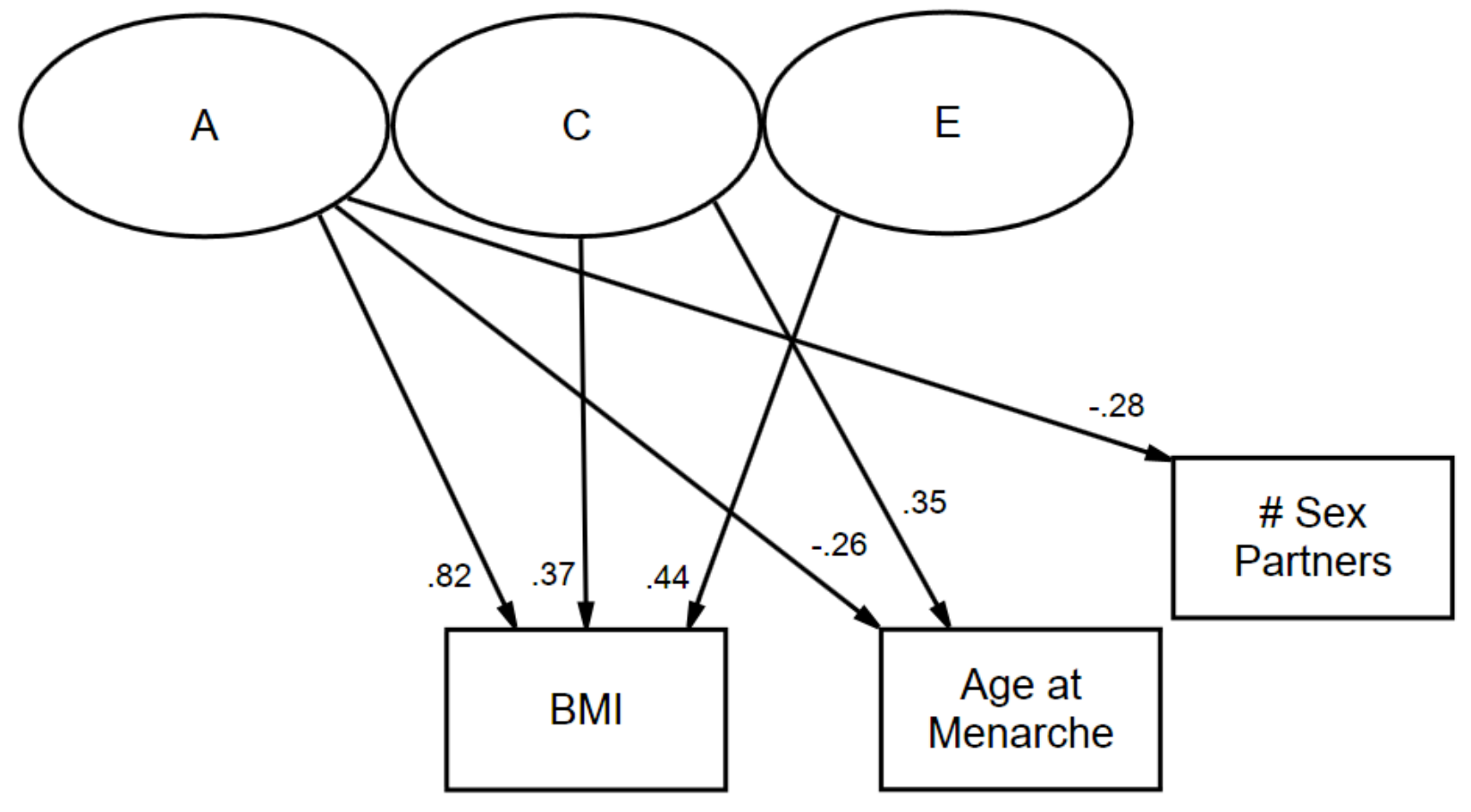

A

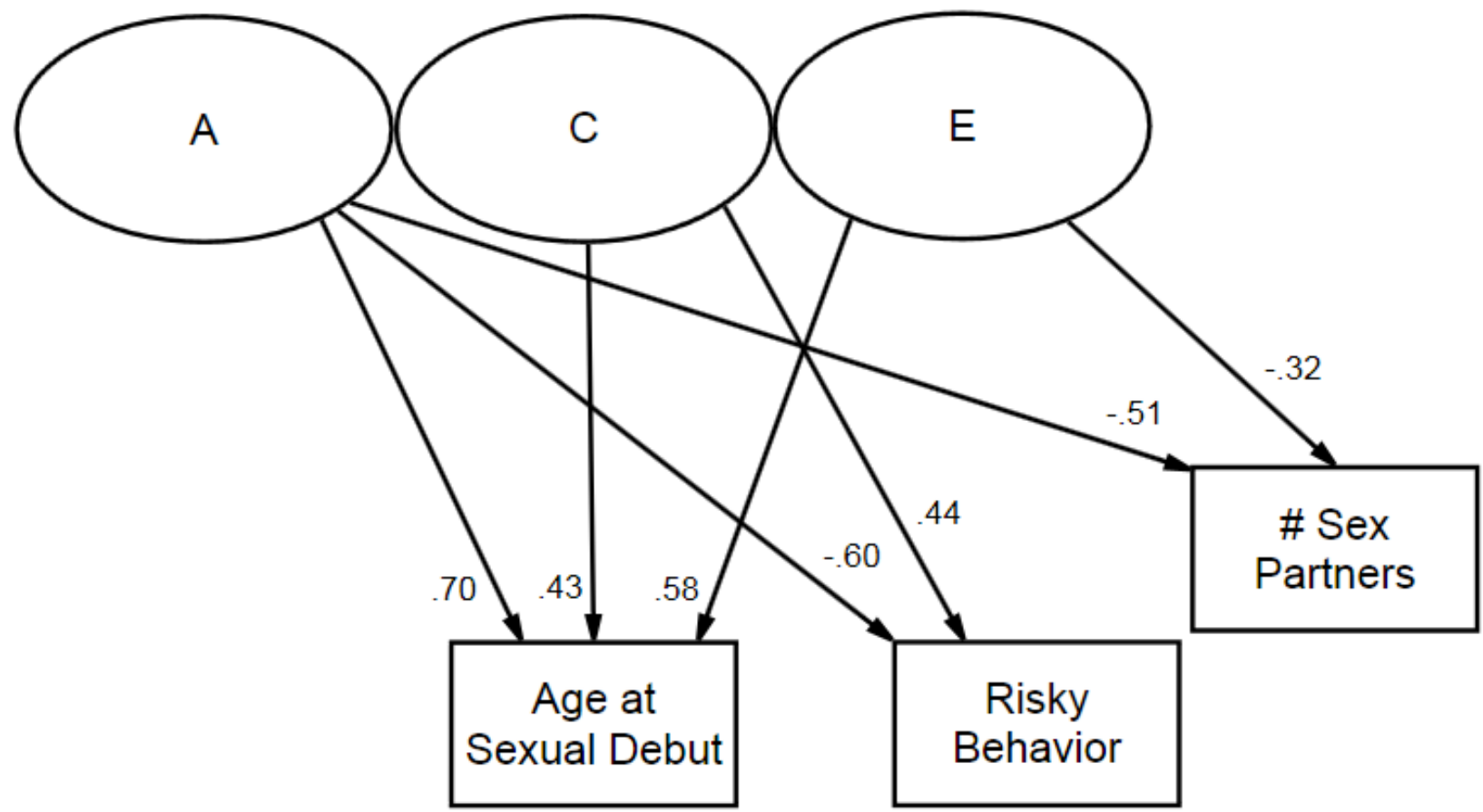

B

Figure 6. (A) Multivariate ACE model of associations among BMI, number of sex partners, and risky behavior. Only significant effects shown. (B) Multivariate ACE model of associations among age at sexual debut, number of sex partners, and risky behavior. Only significant effects shown. 


\section{Supplement to: Assessing Effects of Life History Antecedents on Age at Menarche and Sexual Debut Using a Genetically Informative Design}

\section{Parameter Estimates}

Final SEM Parameter Estimates

\begin{tabular}{|c|c|c|c|c|c|c|c|}
\hline & & & $b$ & SE & $b / \mathrm{SE}$ & $p$ & $\beta$ \\
\hline \multirow[t]{7}{*}{ Environmental Stress } & \multirow[t]{7}{*}{$\rightarrow$} & Divorce/Single & 1.475 & 0.432 & 3.417 & 0.001 & 0.828 \\
\hline & & Early CSA & 0.460 & 0.182 & 2.528 & 0.011 & 0.418 \\
\hline & & Early ACES & 0.289 & 0.097 & 2.966 & 0.003 & 0.195 \\
\hline & & Parent Education & -0.341 & 0.038 & -8.910 & 0.000 & -0.323 \\
\hline & & Income & -0.555 & 0.047 & -11.933 & 0.000 & -0.836 \\
\hline & & Mom public assistance & 1.553 & 0.463 & 3.351 & 0.001 & 0.841 \\
\hline & & Neighborhood quality & -0.229 & 0.082 & -2.806 & 0.005 & -0.230 \\
\hline \multirow[t]{5}{*}{ Age at sexual debut } & \multirow[t]{5}{*}{$\leftarrow$} & Environmental stress & -0.221 & 0.109 & -2.023 & 0.043 & -0.209 \\
\hline & & Age at menarche & -0.010 & 0.079 & -0.130 & 0.896 & -0.010 \\
\hline & & Father absence & -0.116 & 0.075 & -1.545 & 0.122 & -0.109 \\
\hline & & Age & 0.056 & 0.054 & 1.028 & 0.304 & 0.083 \\
\hline & & \# of children & 0.148 & 0.064 & 2.291 & 0.022 & 0.203 \\
\hline \multirow[t]{5}{*}{ Age at menarche } & \multirow[t]{5}{*}{$\leftarrow$} & Environmental stress & -0.075 & 0.087 & -0.870 & 0.384 & -0.074 \\
\hline & & Father absence & 0.083 & 0.083 & 0.990 & 0.322 & 0.080 \\
\hline & & BMI & -0.334 & 0.139 & -2.397 & 0.017 & -0.150 \\
\hline & & Birthweight & 0.044 & 0.067 & 0.662 & 0.508 & 0.050 \\
\hline & & Age & -0.044 & 0.050 & -0.878 & 0.380 & -0.068 \\
\hline \multirow[t]{3}{*}{ \# sex partners } & \multirow[t]{3}{*}{$\leftarrow$} & Age at sexual debut & -0.609 & 0.100 & -6.098 & 0.000 & -0.524 \\
\hline & & Age & 0.005 & 0.053 & 0.095 & 0.925 & 0.006 \\
\hline & & BMI & -0.690 & 0.170 & -4.056 & 0.000 & -0.259 \\
\hline \multirow[t]{2}{*}{ Risky behavior } & \multirow[t]{2}{*}{$\leftarrow$} & Age at sexual debut & -0.290 & 0.067 & -4.327 & 0.000 & -0.290 \\
\hline & & Age & -0.075 & 0.041 & -1.822 & 0.068 & -0.112 \\
\hline \multirow[t]{4}{*}{ BMI } & \multirow[t]{4}{*}{$\leftrightarrow$} & Environmental stress & 0.083 & 0.024 & 3.408 & 0.001 & 0.179 \\
\hline & & Birthweight & 0.038 & 0.040 & 0.944 & 0.345 & 0.07 \\
\hline & & No children & 0.016 & 0.037 & 0.427 & 0.669 & 0.023 \\
\hline & & Father absence & -0.031 & 0.032 & -0.980 & 0.327 & -0.069 \\
\hline \multirow[t]{3}{*}{ Birthweight } & \multirow[t]{3}{*}{$\leftrightarrow$} & Environmental stress & 0.115 & 0.113 & 1.019 & 0.308 & 0.099 \\
\hline & & \# of children & 0.090 & 0.132 & 0.680 & 0.497 & 0.053 \\
\hline & & Father absence & 0.060 & 0.076 & 0.783 & 0.434 & 0.052 \\
\hline \multirow[t]{2}{*}{ \# of children } & \multirow[t]{2}{*}{$\leftrightarrow$} & Environmental stress & 0.233 & 0.120 & 1.951 & 0.051 & 0.161 \\
\hline & & Father absence & -0.129 & 0.068 & -1.878 & 0.06 & -0.090 \\
\hline Father absence & $\leftrightarrow$ & Environmental stress & 0.206 & 0.062 & 3.308 & 0.001 & 0.209 \\
\hline Early ACES & $\leftrightarrow$ & Early CSA & 0.534 & 0.130 & 4.090 & 0.000 & 0.367 \\
\hline
\end{tabular}


\begin{tabular}{llllllll} 
Risky behavior & $\leftrightarrow$ & \# sex partners & 0.395 & 0.066 & 5.975 & 0.000 & 0.395 \\
\hline
\end{tabular}

Notes: \# = number. $\mathrm{SE}=$ standard error. ACES = adverse childhood experiences. CSA = childhood sexual abuse.

$\mathrm{BMI}=$ body mass index.

Bivariate ACE Parameter Estimates

\begin{tabular}{|c|c|c|c|c|c|c|c|}
\hline & & & $b$ & SE & $b / \mathrm{SE}$ & $p$ & $\beta$ \\
\hline \multicolumn{8}{|c|}{ Age at Sexual Debut } \\
\hline A & $\rightarrow$ & Age at sexual debut & 0.851 & - & - & - & 0.699 \\
\hline $\mathrm{C}$ & $\rightarrow$ & Age at sexual debut & 0.515 & - & - & - & 0.423 \\
\hline $\mathrm{E}$ & $\rightarrow$ & Age at sexual debut & 0.703 & - & - & - & 0.577 \\
\hline \multicolumn{8}{|c|}{ Age at Menarche } \\
\hline A & $\rightarrow$ & Age at menarche & 0.756 & - & - & - & 0.675 \\
\hline $\mathrm{C}$ & $\rightarrow$ & Age at menarche & 0.000 & - & - & - & 0.000 \\
\hline $\mathrm{E}$ & $\rightarrow$ & Age at menarche & 0.827 & - & - & - & 0.738 \\
\hline
\end{tabular}

Notes: $\mathrm{A}=$ additive genetic, $\mathrm{C}=$ shared environment, $\mathrm{E}=$ nonshared environment. $\mathrm{SE}=$ standard error.

Multivariate ACE Parameter Estimates

\begin{tabular}{|c|c|c|c|c|c|c|c|}
\hline & & & $b$ & $\mathrm{SE}$ & $b / \mathrm{SE}$ & $p$ & $\beta$ \\
\hline \multicolumn{8}{|c|}{$B M I$} \\
\hline A & $\rightarrow$ & BMI & 0.710 & - & - & - & 0.819 \\
\hline $\mathrm{C}$ & $\rightarrow$ & BMI & 0.320 & - & - & - & 0.369 \\
\hline $\mathrm{E}$ & $\rightarrow$ & BMI & 0.380 & - & - & - & 0.439 \\
\hline A & $\rightarrow$ & \# sex partners & -0.299 & 0.168 & -1.78 & 0.075 & -0.280 \\
\hline $\mathrm{C}$ & $\rightarrow$ & \# sex partners & 0.200 & 0.244 & 0.818 & 0.413 & 0.187 \\
\hline $\mathrm{E}$ & $\rightarrow$ & \# sex partners & 0.084 & 0.126 & 0.667 & 0.505 & 0.079 \\
\hline A & $\rightarrow$ & Age at menarche & -0.288 & 0.234 & -1.229 & 0.219 & -0.259 \\
\hline $\mathrm{C}$ & $\rightarrow$ & Age at menarche & 0.383 & 0.370 & 1.035 & 0.301 & 0.345 \\
\hline $\mathrm{E}$ & $\rightarrow$ & Age at menarche & -0.054 & 0.117 & -0.459 & 0.646 & -0.048 \\
\hline \# sex partners 1 & $\leftrightarrow$ & \# sex partners 2 & 0.465 & 0.112 & 4.158 & 0.000 & 0.465 \\
\hline Age at menarche 1 & $\leftrightarrow$ & Age at menarche 2 & 0.366 & 0.227 & 1.613 & 0.107 & 0.366 \\
\hline \multicolumn{8}{|c|}{ Age at Sexual Debut } \\
\hline A & $\rightarrow$ & Age at sexual debut & 0.851 & - & - & - & 0.699 \\
\hline $\mathrm{C}$ & $\rightarrow$ & Age at sexual debut & 0.515 & - & - & - & 0.425 \\
\hline $\mathrm{E}$ & $\rightarrow$ & Age at sexual debut & 0.703 & - & - & - & 0.577 \\
\hline A & $\rightarrow$ & \# sex partners & -0.643 & 0.254 & -2.535 & 0.011 & -0.509 \\
\hline $\mathrm{C}$ & $\rightarrow$ & \# sex partners & 0.137 & 0.234 & 0.585 & 0.559 & 0.108 \\
\hline $\mathrm{E}$ & $\rightarrow$ & \# sex partners & -0.409 & 0.129 & -3.176 & 0.001 & -0.324 \\
\hline A & $\rightarrow$ & Risky behavior & -0.932 & 0.997 & -0.935 & 0.350 & -0.596 \\
\hline $\mathrm{C}$ & $\rightarrow$ & Risky behavior & 0.694 & 0.965 & 0.719 & 0.472 & 0.444 \\
\hline $\mathrm{E}$ & $\rightarrow$ & Risky behavior & -0.305 & 0.206 & -1.481 & 0.139 & -0.195 \\
\hline \# sex partners 1 & $\leftrightarrow$ & \# sex partners 2 & 0.405 & 0.198 & 2.045 & 0.041 & 0.405 \\
\hline Risky behavior 1 & $\leftrightarrow$ & Risky behavior 2 & -0.522 & 2.057 & -0.254 & 0.800 & -0.522 \\
\hline
\end{tabular}

Notes: $\mathrm{A}=$ additive genetic, $\mathrm{C}=$ shared environment, $\mathrm{E}=$ nonshared environment. $\#=$ number. $\mathrm{BMI}=$ body mass index. $\mathrm{SE}=$ standard error. 
Correlations for Variables in ACE Models

\begin{tabular}{|c|c|c|c|c|c|c|c|c|c|c|c|c|}
\hline & & & 11 & 12 & 21 & 22 & 31 & 32 & 41 & 42 & 51 & 52 \\
\hline \multicolumn{13}{|c|}{$\underline{D Z}$} \\
\hline $\begin{array}{l}\text { Age at } \\
\text { menarche } 1\end{array}$ & 11 & & & .092 & -.096 & -.187 & .119 & .060 & -.000 & -.000 & -.255 & -.000 \\
\hline $\begin{array}{l}\text { Age at } \\
\text { menarche } 2\end{array}$ & 12 & & .484 & & .180 & .038 & -.186 & -.095 & -.100 & -.000 & .100 & -.195 \\
\hline $\begin{array}{l}\text { Age at sexual } \\
\text { debut } 1\end{array}$ & 21 & & .159 & .189 & & .423 & -.483 & -.221 & -.337 & .060 & .000 & .100 \\
\hline $\begin{array}{l}\text { Age at sexual } \\
\text { debut } 2\end{array}$ & 22 & & -.153 & .091 & .667 & & -.230 & -.454 & -.214 & -.189 & -.100 & -.000 \\
\hline $\begin{array}{l}\text { \# sex partners } \\
1\end{array}$ & 31 & $\underline{M Z}$ & . 185 & .084 & -.522 & -.301 & & .349 & .490 & .124 & -.274 & -.100 \\
\hline $\begin{array}{l}\text { \# sex partners } \\
2\end{array}$ & 32 & & .281. & .219 & -.298 & -.427 & .523 & & .211 & .384 & .177 & .156 \\
\hline $\begin{array}{l}\text { Risky } \\
\text { behavior 1 }\end{array}$ & 41 & & .131 & .073 & -.281 & -.298 & .444 & .276 & & .190 & -.033 & -.072 \\
\hline $\begin{array}{l}\text { Risky } \\
\text { behavior } 2\end{array}$ & 42 & & .128 & .073 & -.234 & -.370 & .182 & .505 & .339 & & .239 & .239 \\
\hline BMI 1 & 51 & & -.057 & -.132 & .000 & -.069 & -245 & -.219 & .008 & .000 & & .470 \\
\hline BMI 2 & 52 & & -.017 & .017 & .155 & -.117 & -.126 & -.176 & .085 & -.040 & .797 & \\
\hline
\end{tabular}

Notes: $\mathrm{MZ}$ twin correlations below diagonal and $\mathrm{MZ}$ correlations above. $\mathrm{BMI}=$ body mass index. \# = number. Polychoric correlations estimated for all pairs of ordinal variables. Polyserial correlations estimated for all pairs in one variable is ordinal and the other is continuous. Pearson correlations estimated for the pair of continuous BMI variables.

\section{Model Specification}

We specified bivariate ACE models to decompose the phenotypic variance in age at menarche and age at sexual debut into additive genetic, shared environmental, and nonshared environmental sources. Latent factor means and variances were set to zero and one, respectively. Given that our observed variables had ordered categories (except BMI), latent response variables were mapped onto observed variables with thresholds. Because we used theta parameterization, the residual variance of each observed variable was part of the model and fixed to zero. As is typically the case in twin models, the model was symmetric and parameters were constrained equal between twins in a pair. We incorporated information about zygosity into the MZ portion of the model by constraining additive genetic and shared environmental covariances to one. For 
the DZ portion of the model, we constrained the additive genetic and shared environmental covariances to .5 and one, respectively.

Initially, our models converged but standard errors could not be computed. We resolved this problem by specifying a second set of models in which the thresholds and ACE loadings were fixed to the values initially observed. All model fit information and estimates were available for these models and reported in the text and supplement.

Next, we specified multivariate ACE models to decompose significant effects detected in our full SEM into additive genetic, shared environmental, and nonshared environmental components. As mentioned in the text, multivariate models of this sort are described in Turkheimer and Harden (2014). To decompose the effects of age at sexual debut on number of sex partners and risky behavior, we added the latter two variables to our bivariate ACE model for age at sexual debut and regressed them onto its ACE components. This model was symmetric and parameters were constrained equal between twins in a pair, including the twin 1 and 2 age at sexual debut ACE effects on number of sex partners and risky behavior, as well as the thresholds for the latter two variables. To accommodate potential unique genetic and environmental influences on number of partners and risky behavior, a between twin residual covariance for each was estimated and allowed to vary by zygosity group.

To test a multivariate ACE model for BMI, we first specified a bivariate ACE model using the same specifications as for age at menarche and sexual debut, except intercepts were included in the model instead of thresholds because BMI was continuous. We then added age at menarche and number of sex partners to this model, regressing them onto the BMI ACE components. Like the previous multivariate ACE model, this model was symmetric and parameters were constrained equal between twins in a pair, including the twin 1 and 2 BMI ACE 
effects on age at menarche and number of sex partners, as well as the thresholds for the latter two variables. To accommodate potential unique genetic and environmental influences on age at menarche and number of partners, a between twin residual covariance for each was estimated and allowed to vary by zygosity group.

\title{
Syntax for ACE Models
}

Age at Menarche Bivariate ACE

\section{DATA:}

FILE IS " ";

VARIABLE:

NAMES ARE famid psuscid region aid1 aid2 related ageW1 racewhite 1 racewhite2 raceblack1 bmi1 bmi2 Birthweight1 Birthweight2 NoChildren1 NoChildren2 MarStat1 Marstat2 AgeMenarche1 AgeMenarche2 NoPartners1W3 NoPartners2W3 AgeSexDebut1 AgeSexDebut2 NoVagSex1 NoVagSex 2 NoChildDesire1 NoChildDesire2 RelateDad1 RelateDad2 d_fabsence 1 d_fabsence 2 fabsence_yrs1 fabsence_yrs2 pc8_r_yr1 pc8_r_yr2 fabsence_yrs_pq1 fabsence_yrs_pq2 fabsence_menarche1 fabsence_menarche2 SingleMother Married DivorcedSeparated Early ACES EarlyCSA ParentSES FAIndex Nhood RiskyBx1 RiskyBx2 ParentEd Income DadPAS MomPAS DadR RiskyBx1b RiskyBx2b;

MISSING ARE ALL (-9999);

USEVARIABLES ARE AgeMenarche1 AgeMenarche2;

CATEGORICAL ARE AgeMenarche1 AgeMenarche2;

GROUPING IS related (1=MZ 2=DZ);

DEFINE:

IF related EQ .5 THEN related $=2$;

IF related EQ 1 THEN related $=1$;

\author{
ANALYSIS: \\ MODEL = NOCOVARIANCES; \\ PARAMETERIZATION IS THETA; \\ ESTIMATOR IS WLSMV; \\ ITERATIONS $=1000$; \\ CONVERGENCE $=0.0005$; \\ COVERAGE $=0.10$;
}

OUTPUT: SAMPSTAT RESIDUAL STANDARDIZED TECH5 MODINDICES(0);

MODEL:

A1 BY AgeMenarche1 @.756; 
C1 BY AgeMenarche1@.000;

E1 BY AgeMenarche1@.827;

A2 BY AgeMenarche2@.756;

C2 BY AgeMenarche2@.000;

E2 BY AgeMenarche2@.827;

\section{A1-E2@1;}

[A1-E2@0];

\section{A1 WITH A2@1; \\ C1WITHC2@1;}

[AgeMenarche1\$1@-1.632]; [AgeMenarche2\$1@-1.632];

[AgeMenarche1\$2@-0.933]; [AgeMenarche2\$2@-0.933];

[AgeMenarche1\$3@-0.092]; [AgeMenarche2\$3@-0.092];

[AgeMenarche1\$4@0.595]; [AgeMenarche2\$4@0.595];

[AgeMenarche1\$5@1.222]; [AgeMenarche2\$5@1.222];

[AgeMenarche1\$6@1.602]; [AgeMenarche2\$6@1.602];

[AgeMenarche1\$7@2.096]; [AgeMenarche2\$7@2.096];

Model MZ:

AgeMenarche1@0;

AgeMenarche2@0;

Model DZ:

A1 WITHA2@.5;

AgeMenarche1@0;

AgeMenarche2@0;

Age at Sexual Debut Bivariate ACE

DATA:

FILE IS " ";

VARIABLE:

NAMES ARE famid psuscid region aid1 aid2 related ageW1 racewhite 1 racewhite 2 raceblack1 bmi1 bmi2 Birthweight1 Birthweight2 NoChildren1 NoChildren2 MarStat1 Marstat2 AgeMenarche1 AgeMenarche2 NoPartners1W3 NoPartners2W3 AgeSexDebut1 AgeSexDebut 2 NoVagSex1 NoVagSex 2 NoChildDesire1 NoChildDesire2 RelateDad1 RelateDad2 d_fabsence1 d_fabsence 2 fabsence_yrs1 fabsence_yrs2 pc8_r_yr1 pc8_r_yr2 fabsence_yrs_pq1 fabsence_yrs_pq2 fabsence_menarche1 fabsence_menarche2 SingleMother Married DivorcedSeparated Early ACES EarlyCSA ParentSES FAIndex Nhood RiskyBx1 RiskyBx2 ParentEd Income DadPAS MomPAS DadR RiskyBx1b RiskyBx2b;

MISSING ARE ALL (-9999);

USEVARIABLES ARE AgeSexDebut1 AgeSexDebut2; CATEGORICAL ARE AgeSexDebut1 AgeSexDebut2; 
GROUPING IS related (1=MZ 2=DZ);

DEFINE:

IF related EQ .5 THEN related $=2$;

IF related EQ 1 THEN related $=1$;

\author{
ANALYSIS: \\ MODEL = NOCOVARIANCES; \\ PARAMETERIZATION IS THETA; \\ ESTIMATOR IS WLSMV; \\ ITERATIONS $=1000$; \\ CONVERGENCE $=0.0005$; \\ COVERAGE $=0.10$
}

OUTPUT: SAMPSTAT RESIDUAL STANDARDIZED TECH5 MODINDICES(0);

MODEL:

A1 BY AgeSexDebut1@.851;

C1 BY AgeSexDebut1@.515;

E1 BY AgeSexDebut1@.703;

A2 BY AgeSexDebut2@.815;

C2 BY AgeSexDebut2@.515;

E2 BY AgeSexDebut2@.703;

\begin{abstract}
[A1-E2@0];
A1-E2@1;

[AgeSexDebut1\$1@-1.790]; [AgeSexDebut2\$1@-1.790];

[AgeSexDebut1\$2@-1.284]; [AgeSexDebut2\$2@-1.284];

[AgeSexDebut1\$3@-0.743]; [AgeSexDebut2\$3@-0.743];

[AgeSexDebut1\$4@-0.252]; [AgeSexDebut2\$4@-0.252];

[AgeSexDebut1\$5@0.267]; [AgeSexDebut2\$5@0.267];

[AgeSexDebut1\$6@0.826]; [AgeSexDebut2\$6@ 0.826];

[AgeSexDebut1\$7@1.098]; [AgeSexDebut2\$7@ 1.098];

[AgeSexDebut1\$8@1.383]; [AgeSexDebut2\$8@1.383];
\end{abstract}

\title{
A1 WITH A2@1; \\ C1WITHC2@1; \\ Model MZ: \\ AgeSexDebut1@0; \\ AgeSexDebut2@0;
}

Model DZ:

A1 WITH A2@.5;

AgeSexDebut1@0;

AgeSexDebut2@0; 
DATA:

FILE IS " ";

VARIABLE:

NAMES ARE famid psuscid region aid1 aid2 related ageW1 racewhite 1 racewhite2 raceblack1 bmi1 bmi2 Birthweight1 Birthweight2 NoChildren1 NoChildren2 MarStat1 Marstat2 AgeMenarche1 AgeMenarche2 NoPartners1W3 NoPartners2W3 AgeSexDebut1 AgeSexDebut 2 NoVagSex1 NoVagSex 2 NoChildDesire1 NoChildDesire2 RelateDad1 RelateDad2 d_fabsence 1 d_fabsence 2 fabsence_yrs1 fabsence_yrs2 pc8_r_yr1 pc8_r_yr2 fabsence_yrs_pq1 fabsence_yrs_pq2 fabsence_menarche1 fabsence_menarche2 SingleMother Married DivorcedSeparated Early ACES EarlyCSA ParentSES FAIndex Nhood RiskyBx1 RiskyBx2 ParentEd Income DadPAS MomPAS DadR RiskyBx1b RiskyBx2b;

MISSING ARE ALL (-9999);

USEV ARIABLES ARE AgeSexDebut 1 AgeSexDebut2 NoVagSex1 NoVagSex2 RiskyBx1b RiskyBx2b;

CATEGORICAL ARE AgeSexDebut1 AgeSexDebut2 NoVagSex 1 NoVagSex2 RiskyBx $1 b$ RiskyBx2b;

GROUPING IS related (1=MZ 2=DZ);

DEFINE:

IF related EQ .5 THEN related $=2$;

IF related EQ 1 THEN related $=1$;

ANALYSIS:

MODEL = NOCOVARIANCES;

PARAMETERIZATION IS THETA;

ESTIMATOR IS WLSMV;

ITERATIONS $=1000$;

CONVERGENCE $=0.0005$;

COVERAGE $=0.10$;

OUTPUT: SAMPSTAT RESIDUAL STANDARDIZED TECH5 MODINDICES(0);

MODEL:

A1 BY AgeSexDebut1@.85;

C1 BY AgeSexDebut1@.52;

E1 BY AgeSexDebut1@.70;

A2 BY AgeSexDebut2@.85;

C2 BY AgeSexDebut2@.52;

E2 BY AgeSexDebut2@.70;

[A1-E2@0];

A1-E2@1;

[AgeSexDebut1\$1@-1.790]; [AgeSexDebut2\$1@-1.790]; 
[AgeSexDebut1\$2@-1.284]; [AgeSexDebut2\$2@-1.284];

[AgeSexDebut1\$3@-0.743]; [AgeSexDebut2\$3@-0.743];

[AgeSexDebut1\$4@-0.252]; [AgeSexDebut2\$4@-0.252];

[AgeSexDebut1\$5@0.267]; [AgeSexDebut2\$5@0.267];

[AgeSexDebut1\$6@0.826]; [AgeSexDebut2\$6@ 0.826];

[AgeSexDebut1\$7@1.098]; [AgeSexDebut2\$7@ 1.098];

[AgeSexDebut1\$8@1.383]; [AgeSexDebut2\$8@1.383];

NoVagSex 1 ON A1(12);

NoVagSex 1 ON C1(13);

NoVagSex 1 ON E1(14);

[NoVagSex 1\$1-NoVagSex1\$9](20-28);

NoVagSex2 ON A2(12);

NoVagSex2 ON C2(13);

NoVagSex 2 ON E2(14);

[NoVagSex2\$1-NoVagSex2\$9](20-28);

RiskyBx1b ON A1(15);

RiskyBx1b ON C1(16);

RiskyBx1b ON E1(17);

[RiskyBx1b\$1-RiskyBx1b\$3](40-42);

RiskyBx2b ON A2(15);

RiskyBx2b ON C2(16);

RiskyBx2b ON E2(17);

[RiskyBx2b\$1-RiskyBx2b\$3](40-42);

A1 WITHA2@1;

C1 WITHC2@1;

Model MZ:

AgeSexDebut1@0;

AgeSexDebut2@0;

NoVagSex 1 WITH NoVagSex2;

RiskyBx1b WITH RiskyBx2b;

Model DZ:

A1 WITH A2@.5;

AgeSexDebut1@0;

AgeSexDebut2@0;

NoVagSex 1 WITH NoVagSex2;

RiskyBx1b WITH RiskyBx2b; 
DATA:

FILE IS " ";

VARIABLE:

NAMES ARE famid psuscid region aid1 aid2 related ageW1 racewhite 1 racewhite2 raceblack1 bmi1 bmi2 Birthweight1 Birthweight2 NoChildren1 NoChildren2 MarStat1 Marstat2 AgeMenarche1 AgeMenarche2 NoPartners1W3 NoPartners2W3 AgeSexDebut1 AgeSexDebut 2 NoVagSex1 NoVagSex 2 NoChildDesire 1 NoChildDesire2 RelateDad1 RelateDad2 d_fabsence 1 d_fabsence 2 fabsence_yrs1 fabsence_yrs2 pc8_r_yr1 pc8_r_yr2 fabsence_yrs_pq1 fabsence_yrs_pq2 fabsence_menarche1 fabsence_menarche2

SingleMother Married DivorcedSeparated Early ACES EarlyCSA ParentSES FAIndex Nhood RiskyBx1 RiskyBx2 ParentEd Income DadPAS MomPAS DadR RiskyBx1b RiskyBx2b;

MISSING ARE ALL (-9999);

USEVARIABLES ARE bmi1 bmi2 NoVagSex1 NoVagSex2 AgeMenarche1 AgeMenarche2;

CATEGORICAL ARE NoVagSex1 NoVagSex2 AgeMenarche1 AgeMenarche2;

GROUPING IS related (1=MZ 2=DZ);

DEFINE:

IF related EQ .5 THEN related $=2$;

IF related EQ 1 THEN related $=1$;

ANALYSIS:

MODEL = NOCOVARIANCES;

PARAMETERIZATION IS THETA;

ESTIMATOR IS WLSMV;

ITERATIONS $=1000$;

CONVERGENCE $=0.0005$;

COVERAGE $=0.10$;

OUTPUT: SAMPSTAT RESIDUAL STANDARDIZED TECH5 MODINDICES(0);

MODEL:

A1 BY bmi1@.71;

C1 BY bmi1@.32;

E1 BY bmi1@.38;

[bmi1](4);

A2 BY bmi2@.71;

C2 BY bmi2@.32;

E2 BY bmi2@.38;

[bmi2](4);

[A1-E2@0];

A1-E2@1; 
NoVagSex 1 ON A1(12);

NoVagSex 1 ON C1(13);

NoVagSex 1 ON E1(14);

[NoVagSex 1\$1-NoVagSex1\$9](20-28);

NoVagSex 2 ON A2(12);

NoVagSex 2 ON C2(13);

NoVagSex2 ON E2(14);

[NoVagSex2\$1-NoVagSex2\$9](20-28);

AgeMenarche1 ON A1(15);

AgeMenarche1 ON C1(16);

AgeMenarche1 ON E1(17);

[AgeMenarche1\$1-AgeMenarche 1\$7](40-46);

AgeMenarche2 ON A2(15);

AgeMenarche2 ON C2(16);

AgeMenarche2 ON E2(17);

[AgeMenarche2\$1-AgeMenarche2\$7](40-46);

A1 WITH A2@1;

C1 WITHC2@1;

Model MZ:

bmi1@0;

bmi2@0;

NoVagSex 1 WITH NoVagSex2;

AgeMenarche1 WITH AgeMenarche2;

Model DZ:

A1 WITH A2@.5;

bmi1@0;

bmi2@0;

NoVagSex 1 WITH NoVagSex2;

AgeMenarche1 WITH AgeMenarche2; 


\section{List of Footnotes}

1. Although oversampled, prior research has shown that the twin subsample of the Add Health does not differ substantially from the nonsibling sample on a variety of measures (e.g., Barnes \& Boutwell, 2013; Jacobson \& Rowe, 1998; Nedelec et al., 2017).

2. Our preregistered sample size included 254 twin pairs. We ultimately elected to include data from three additional twins with no co-twin data, bringing the number of pairs to 257.

3. These were, "how often in the last month have you (parent) had five or more drinks on one occasion" (PA62), "how many other states or countries have you lived since June 1995" (H3HR20), "since the beginning of June 1995 at how many (other) addresses have you lived" (H3HR18).

4. Specifically, $60.5 \%$ were raised by biological fathers, $14.5 \%$ were raised by unrelated father figures, and less than $6.0 \%$ were raised by any type of non-biological but related father figure (i.e., grandfather, brother, uncle, or other male relative.)

5. Missing values were saved for participants who were missing data on the indicators analyzed with PCA. Missing data were handled via pairwise present in the SEM analyses.

6. Age of menarche at Wave 2 [H2FP4] was employed to reduce missingness; additionally, respondents who reached age of menarche prior to father absence were coded as " 0 " on this item

7. We planned to use robust maximum likelihood (MLR) as the estimator but we had a mix of continuous and categorical indicators and needed to specify residual covariances between the latter. This can only be accomplished via mixture modeling in the context of MLR and we found this approach much more computationally intensive than WLSMV. We preregistered the decision to use WLSMV if MLR was too computationally intensive.

8. The Wald $z$-tests and global $\chi^{2}$ test are only asymptotically equivalent, meaning that they will only match closely in large samples. Wald tests are regarded as a poor choice to testing hypotheses in samples of modest size, where second-order parameters converge slowly to normality. Additionally, the Wald $z$-test is sensitive to the choice of parameterization, whereas the global $\chi^{2}$ test is invariant to change-of-parameter. 